\title{
Wellposedness and Analytic Smoothing Effect for the Benjamin-Ono Equation
}

By

Elena KaIKInA, Keiichi Kato,* Pavel I. NAumkin*** and Takayoshi OGaWA ${ }^{\dagger}$

\section{§1. Introduction}

We study smoothing effect for the following nonlinear dispersive equation of the Benjamin-Ono type:

$$
\left\{\begin{array}{l}
\partial_{t} u+\mathcal{H}_{x} \partial_{x}^{2} u+\partial_{x} u^{2}=0, \quad t \in(-T, T), \quad x \in \mathbb{R}, \\
u(0, x)=\phi(x),
\end{array}\right.
$$

where $u(t, x): \mathbb{R} \times \mathbb{R} \rightarrow \mathbb{R}$ is unknown function and $\mathcal{H}_{x}$ denotes the Hilbert transform defined by $\mathcal{H}_{x} v=\mathcal{F}(\xi /(i|\xi|)) \hat{v}$. This equation arises in the water wave theory and $u$ describes long internal gravity wave in deep stratified fluid (see [2], [42]). Our problem here is to investigate a sufficient condition of initial data $\phi$ on which the solution has regularizing property up to analyticity.

The existence and well-posedness problem of this equation is studied by many authors. We refer to T. Kato [29], Iorio Jr. [22], Ponce [43], Kenig, Ponce and Vega [35] and reference therein. In the recent progress for the nonlinear dispersive equations, large amounts of studies are based on the smoothing effect. When we consider the well-posedness of those type of equation, $L^{2}$ based (Sobolev) space is usually considered and the same order of the regularity for solutions is derived as the initial data $\phi$. Concerning the dispersive equation such as KdV, nonlinear Schrödinger and the Benjamin-Ono type equations, local or restricted version of smoothing effect (in terms of weighted norm) was

Communicated by H. Okamoto. Recieved May 21, 2001. Revised November 16, 2001.

AMS Subject Classification(s): 35Q53.

* Departamento de Ciencias Baiscas, Istituto Tecnologico de Morelia, CP58120, Morelia, Michoacan, Mexico.

** Department of Mathematics, Tokyo University of Science, Shinjyuku-ku, Tokyo 162-8601, Japan.

*** Instituto de Fisica Matematicas, Universidad Michoacana, CP58040, Morelia, Michoacan, Mexico.

${ }^{\dagger}$ Graduate School of Mathematics, Kyushu University, Fukuoka 812-8581, Japan. 
observed. As the most well understood case, we refer to the results of nonlinear Schrödinger equations, [4], [6], [7], [12], [14], [15], [16], [18], [19], [26], [28], [41] and that of linear Schrödinger equations in [9], [24] and [44]. Since the Benjamin-Ono equation has a similar dispersive structure to the Schrödinger equations, we expect that analogous results may hold for the nonlinear problem (1.1).

Concerning analytic smoothing effect, we showed that for a weak initial data including the Dirac delta measure, the corresponding weak solution gains the regularity to analytic in both space and time variables by a method of the conormal vector fields (see K. Kato and Ogawa [25] and also for the Schrödinger cases [26] and K. Kato and Taniguchi [28]). In this paper, we extend these results to the Benjamin-Ono case (1.1). Our method is based on the analogous operator method which is based on the common linear structure to the KdV equation and nonlinear Schrödinger equations: We introduce the generator of space-time dilation $P=2 t \partial_{t}+x \partial_{x}$ that plays a compensating role on which the main linear operator $L=\partial_{t}+\mathcal{H}_{x} \partial_{x}^{2}$ can not gain the regularity. As a consequence, we observe analytic smoothing effect for the solution to (1.1) with the initial data having a singularity at the origin. We state our result more specifically. Let $H^{s}=H^{s}(\mathbb{R})$ be the Sobolev space of order $s$ defined by

$$
H^{s}(\mathbb{R})=\left\{f \in \mathcal{S}^{\prime}:\|f\|_{H^{s}}<\infty\right\}, \quad\|f\|_{H^{s}} \equiv\left\|\langle\xi\rangle^{s} \hat{f}\right\|_{2},
$$

where $\hat{f}=\mathcal{F} f$ denotes the Fourier transform of $f$ and $\langle\cdot\rangle=\left(1+|\cdot|^{2}\right)^{1 / 2} \cdot L_{s}^{2}(\mathbb{R})$ denotes the weighted $L^{2}$ defined by

$$
\|f\|_{L_{s}^{2}} \equiv\left\|\langle x\rangle^{s} f\right\|_{2}<\infty
$$

Theorem 1.1. Let $s>3 / 2$. Suppose that for some $A_{0}>0$, the initial data $\phi \in H^{s}(\mathbb{R})$ and satisfies

$$
\sum_{k=0}^{\infty} \frac{A_{0}^{k}}{((k-1) !)^{2}}\left(\left\|\left(x \partial_{x}\right)^{k} \phi\right\|_{H^{s}}^{2}+\left\|\left(x \partial_{x}\right)^{k} \phi\right\|_{L_{s}^{2}}^{2}\right)<\infty
$$

(where $0 !,(-1) !=1)$. Then there exist positive constants $T, A$ and a unique solution $u \in C\left([-T, T], H^{s}\right)$ of the initial value problem (1.1) such that

$$
\left|\partial_{t}^{j} \partial_{x}^{l} u(t, x)\right| \leq C\left\langle t^{-1}\right\rangle^{j+l}\langle x\rangle^{2 l+3 j} A^{j+l}(j+l) !
$$

valids for any $(t, x) \in([-T, T] \backslash\{0\}) \times \mathbb{R}$ and for any $j, l \in \mathbb{N}$. Namely $u(t, \cdot)$ is a real analytic function in both space and time variables for $t \in[-T, T] \backslash\{0\}$. 
Remark 1. Typical example of the initial data satisfying the assumption above is, for instance, $e^{-|x|^{\alpha}}$ with $\alpha>1,\langle x\rangle^{-\beta}$ with $\beta>2$ or $|x|^{\gamma_{1}}\langle x\rangle^{-\gamma_{2}}$ with $\gamma_{1}>1$ and $\gamma_{2}-\gamma_{1}>2$. In fact, the assumption on the initial data implies that the data have to be analytic except $x=0$. On this point, the data may possibly have $H^{s}$ singularity. Hence the above theorem states that this singularity disappears after time passed. The weakness of this singularity on the data is depending on the space where we may establish the well-posedness of the equation.

The existence and uniqueness result of the Benjamin-Ono equation can be found in the articles by Saut [46], Iorio Jr. [22], Ponce [43]. The global well-posedness in time is also discussed by Kenig-Ponce-Vega [35]. Our result is based on those well-posedness results in the Sobolev space $H^{s}(\mathbb{R})$ with $s>3 / 2$. We should note that the critical well-posedness result on $H^{3 / 2}(\mathbb{R})$ due to Ponce [43] is not covered by our result, since we consider slightly complicated system of the Benjamin-Ono type equations (see the equation (2.2) with (2.1) in Section 2 below) and for this system, the local well-posedness is not well understood in $H^{3 / 2}(\mathbb{R})$. In general, it seems that even the well-posedness for the single Benjamin-Ono equation (1.1) in a weaker space than $H^{3 / 2}$ is not yet established so far as authors know. If this is improved into the system we treat here in $H^{s}(\mathbb{R})$ with $s<3 / 2$, we may extend our result into such a weak space even negative exponent Sobolev spaces. See [25] for this direction of the KdV equation.

It is also known by Iorio Jr. [22] that there is no non-trivial solution in the smaller space than $H^{4}(\mathbb{R}) \cap L_{4}^{2}(\mathbb{R}$ ) (in fact, the threshold seems to be $\left.H^{7 / 2}(\mathbb{R}) \cap L_{7 / 2}^{2}(\mathbb{R})\right)$. In our assumption, the initial data has a strong restriction by a weight for its derivatives. However the weight on the solution itself is just $s>3 / 2$ and this does not contradict the non-existence result by Iorio Jr.

Our argument to gain the regularity for the Benjamin-Ono equation (1.1), is based on the conormal method. This idea is also considered by Hayashi, K. Kato and Ozawa [17]. They considered a weight $\left(x \partial_{x}\right)^{m} m=2,3,4$ and obtained a smoothing effect for a solution to (1.1).

Quite recently, a remarkable ill-posedness result was obtained by Molinet, Saut and Tzvetkov [40], where they proved that the iteration scheme from the integral equation can not yield the well-posedness in any order of the Sobolev spaces. Since our well-posedness result is based on a quadratic form and multiplication with integration by parts, it is possible to avoid this deficiency.

Remark 2. It is well-known that the global in time solution has been obtained to Benjamin-Ono type equations by both the inverse scattering and 
analytical (continuing) methods. One may show that the result holds globally in time. We refer the work by T. Kato and Masuda [31] for this direction of the global analyticity.

By the similar argument as in Theorem 1.1, one can also show the following weaker theorem in the Gevrey and analytic regularity.

Theorem 1.2. Let $s>3 / 2$. Suppose that for some $A_{0}>0$, the initial data $\phi \in H^{s}(\mathbb{R})$ and satisfies

$$
\sum_{k=0}^{\infty} \frac{A_{0}^{k}}{((k-1) !)^{4}}\left(\left\|\left(x \partial_{x}\right)^{k} \phi\right\|_{H^{s}}^{2}+\left\|\left(x \partial_{x}\right)^{k} \phi\right\|_{L_{s}^{2}}^{2}\right)<\infty
$$

(as before $0 !,(-1) !=1)$. Then there exist a positive constant $T$ and a unique solution $u \in C\left([-T, T], H^{s}\right)$ of the initial value problem (1.1) such that $u(t, x)$ is an analytic function in space variable $x$ and a Gevrey class function of order 2 in time variable $t$ for any $(t, x) \in([-T, T] \backslash\{0\}) \times \mathbb{R}$.

Remark 3. In both Theorems, the assumption on the initial data implies the analyticity and Gevrey 2 regularity except the origin respectively. In this sense, these results state that the singularity at the origin immediately disappears after $t>0$ or $t<0$ up to analyticity.

Some related results are obtained for linear and nonlinear Schrödinger equations. For linear variable coefficient case, see Kajitani and Wakabayashi [24], Robbiano and Zuily [44] and for nonlinear case, Chihara [5]. They give a global weighted uniform estimates of the solution with arbitrary order derivative in space variable.

The essential difference in proving the above results from the case for the nonlinear Schrödinger or KdV equation is mainly due to the appearance of the nonlocal operator $\mathcal{H}_{x}$.

Besides, since our method is depending on the commutator argument using the generator of the dilation, we reduce the equation into the infinitely many system of the Benjamin-Ono type equation. Then there appears a difficulty to prove the local well-posedness for those system. It is well known that the Kato type method for the quasi-linear equation ([29]) saves the derivative loss if it is a single equation or has a special structure for the quadratic nonlinearity. However the reduced system here does not seem to have this nice structure. To fill this gap, we invoke the local smoothing property for the linear dispersive equations. This nature for the dispersive equations was observed by several authors 
[39], [30], [48], [53], [18] and [6]. On the other hand, some gauge invariance for the Schrödinger equation also works to save the derivative loss. Hayashi [13] firstly utilized the nonlinear gauge transform to obtaining the existence theorem for the nonlinear Schrödinger equation with derivative nonlinearity. The linear case was studied by Doi [9] and his method was applied to the nonlinear case by Chihara [4] and developed further by Kenig-Ponce-Vega [37]. At this point, the method of the gauge transform and Kato-Mizohata type local smoothing argument was treated as in the unified way and applied into some other dispersive equations cf. [19] and [14]. Here we apply those local smoothing property under the weight condition to save the derivative loss for on the system of equations.

To prove the solution is real analytic in space and time directions, we use some localization technique. Then it is required to treat the non local term carefully to show the higher regularity. We then introduce a weight function which has an explicit commuting estimate with $\mathcal{H}_{x}$. This enables us to handle the nonlocal term $\mathcal{H}_{x}$ in the linear part of the equation. In the following section, we first show the outline of our method and what is the difficulty. Then in the subsequent section we give the local wellposedness of the reduced equations and regularity upto the analyticity in space time directions.

Since the whole argument is rather long, we summarize our main idea and key method in Section 2. In Section 3, we show some useful commutation estimates of the Hilbert transform and the fractional derivative and in Section 4 , we establish the time local well-posedness of the system of the BenjaminOno type equations (2.2). In Section 5 , we show the analyticity of the solution stated in the main theorem by a bootstrap argument as is seen in a sequence of propositions.

We use the following notations: $\langle\cdot\rangle=\left(1+|\cdot|^{2}\right)^{1 / 2} \cdot \mathcal{H}_{x}$ is the Hilbert transform and $D_{x}$ denotes the self adjoint operator $\mathcal{H}_{x} \partial_{x} \cdot\|\cdot\|_{p}$ is the $L^{p}(\mathbb{R})$ norm. $W^{s, p}=W^{s, p}(\mathbb{R})$ is the Sobolev space defined by $\left\|\left\langle D_{x}\right\rangle^{s} f\right\|_{p}<\infty$ and we denote $H^{s}=W^{s, 2}$. The homogeneous Sobolev space $\dot{H}^{s}$ is also defined by $\left\|D_{x}^{s} f\right\|_{2}<\infty . L_{s}^{2}=L_{s}^{2}(\mathbb{R})$ is the weighted $L^{2}$ space given by $\left\|\langle x\rangle^{s} f\right\|_{2}<\infty$. Let $L=\partial_{t}+\mathcal{H}_{x} \partial_{x}^{2}$ be the linear part of the Benjamin-Ono equation and $P=2 t \partial_{t}+x \partial_{x}$ be the dilation operator associated with $L$. For operators $A$ and $B,[A, B]$ stands for the commutator $A B-B A$. Various norm and semi-norm in the Banach space $\mathrm{X}$ is denoted as $\|\cdot\|_{X}$ except $\|\cdot\|_{p}$ for the case $X=L^{p}(\mathbb{R})$. The abbreviated summation $\sum_{k=k_{0}+k_{1}+k_{2}}$ denotes the summation over $k_{0}, k_{1}$ and $k_{2}$ under $0 \leq k_{j} \leq k$ with $k=k_{0}+k_{1}+k_{2}$. The factorial of zero and negative number is always considered as 1 . Similarly, to avoid any 
complicated expression, we regard $k$ and $k_{j}(j=0,1,2)$ as $\max (k, 1), \max \left(k_{j}, 1\right)$ respectively in the notation such as

$$
\sum_{k=k_{0}+k_{1}+k_{2}} \frac{k}{k_{0} ! k_{1} k_{2}} \cdots
$$

\section{§. Method}

In this section we give an overview of the whole proof and present some difference from the proof of the former cases in [25] and [26].

Firstly, as is found in the work by Hayashi, K. Kato and Ozawa [17], we introduce the generator of the dilation $P=2 t \partial_{t}+x \partial_{x}$ corresponding to the linear part of the dispersive equation. Since the commuting relation with the linear dispersive operator $L \equiv \partial_{t}+\mathcal{H}_{x} \partial_{x}^{2}$ is

$$
[L, P]=2 L,
$$

it follows

$$
\begin{aligned}
L P^{k} & =(P+2)^{k} L, \\
(P+2)^{k} \partial_{x} & =\partial_{x}(P+1)^{k}, \quad k=1,2, \ldots .
\end{aligned}
$$

Applying $P^{k}$ to the equation,

$$
\partial_{t}\left(P^{k} u\right)+\mathcal{H}_{x} \partial_{x}^{2}\left(P^{k} u\right)=(P+2)^{k} L u=-(P+2)^{k} \partial_{x}\left(u^{2}\right) .
$$

Then if we set $u_{k}=P^{k} u$ and $B_{k}(u, u)=-(P+2)^{k} \partial_{x} u^{2}$,

$$
\begin{aligned}
(P+1)^{l} u & =(P+1)^{l-1} P u+(P+1)^{l-1} u=\cdots \\
& =\sum_{j=0}^{l} \frac{l !}{j !(l-j) !} P^{j} u
\end{aligned}
$$

and hence

$$
\begin{aligned}
B_{k}(u, u) & =-(P+2)^{k} \partial_{x}\left(u^{2}\right)=-\partial_{x}(P+1)^{k}\left(u^{2}\right) \\
& =-\partial_{x} \sum_{l=0}^{k}\left(\begin{array}{l}
k \\
l
\end{array}\right)(P+1)^{l} u P^{k-l} u \\
& =-\partial_{x} \sum_{l=0}^{k} \sum_{j=0}^{l}\left(\begin{array}{l}
k \\
l
\end{array}\right)\left(\begin{array}{l}
l \\
j
\end{array}\right) P^{j} u P^{k-l} u
\end{aligned}
$$




$$
=-\partial_{x} \sum_{k=k_{0}+k_{1}+k_{2}} \frac{k !}{k_{0} ! k_{1} ! k_{2} !} u_{k_{1}} u_{k_{2}} .
$$

The important point is that the nonlinear terms $B_{k}(u, u)$ maintain the bilinear structure similar to the original Benjamin-Ono equation. This is due that the Leibniz law can be applicable for an operation of $P$. Thus each of $u_{k}$ satisfies the following system of equations;

$$
\left\{\begin{array}{l}
\partial_{t} u_{k}+\mathcal{H}_{x} \partial_{x}^{2} u_{k}=B_{k}(u, u), \quad t, x \in \mathbb{R}, \\
v_{k}(0, x)=\left(x \partial_{x}\right)^{k} \phi(x) .
\end{array}\right.
$$

Therefore, we consider the following system of dispersive equation and show the well-posedness of the system as well as establishing an estimate for the derivatives

$$
\left\{\begin{array}{l}
\partial_{t} u_{k}+\mathcal{H}_{x} \partial_{x}^{2} u_{k}=B_{k}(u, u), \quad t, x \in \mathbb{R}, \\
u_{k}(0, x)=\phi_{k}(x) .
\end{array}\right.
$$

One difficulty to establish the local well-posedness to the above system is that the method of quasi-linear equation by T. Kato [29] does not work well since some of the nonlinear term, say $\partial_{x}\left(u_{k_{1}} u_{k_{2}}\right)$ does not contain the same function $u_{k}$ which represents the principal linear part. Therefore it is required to use some kind of smoothing effect from the dispersive property to avoid the derivative loss. Following the argument due to [4], [19] and [14], we consider a variation of the weight function $E_{x}(x)=\exp \left(-\int_{-\infty}^{x} \omega^{2}(y) d y\right)$, where $\omega(x)$ is a properly chosen weight function. By commuting $E_{x}$ with the linear part, this weight function gains one half derivative under some weight condition, then the local existence and wellposedness for the following infinitely coupled system of Benjamin-Ono type is proved in a proper weighted Sobolev space: We also note that the solution is constructed in a slightly stringent function class such as

$$
\sum_{k=0}^{\infty} \frac{A_{1}^{k}}{(k-1) !^{2}}\left\|u_{k}\right\|_{H^{s} \cap L_{s}^{2}}^{2}<\infty
$$

than the case of the KdV equation (cf. [25]), since we construct the solution via the quadratic argument. Then taking $\phi_{k}=\left(x \partial_{x}\right)^{k} \phi(x)$, the uniqueness and local well-posedness allow us to say $u_{k}=P^{k} u$ for all $k=0,1, \ldots$

Through the process of proving the existence and uniqueness, we obtain the estimate

$$
\left\|P^{k} u\right\|_{H^{s}} \leq C A^{k} k !
$$


Then we would derive the point-wise derivative estimate by using the equation:

$$
\mathcal{H}_{x} \partial_{x}^{2} P^{k} u=-\frac{1}{2 t} P^{k+1} u+\frac{1}{2 t} x \partial_{x} P^{k} u+B_{k}(u, u) .
$$

To treat the second term of the right hand side of (2.4), we employ the localization argument. By a suitable decaying function, we can show that

$$
\left\|a \partial_{x}^{l} P^{k} u(t)\right\|_{H^{1}(\mathbb{R})} \leq C\left\langle t^{-1}\right\rangle^{l} A^{k+l}(k+l) !, \quad k, l=0,1,2, \ldots .
$$

and then by iterative argument, we can shift from the estimate with the operator $P^{k}$ to $\left(t \partial_{t}\right)^{l}$ and conclude

$$
\left\|\left(t \partial_{t}\right)^{l_{1}} \partial_{x}^{l_{2}} u(t)\right\|_{L^{\infty}\left(x_{0}-\delta, x_{0}+\delta\right)} \leq C\left\langle t^{-1}\right\rangle^{l_{1}+l_{2}}\left\langle x_{0}\right\rangle^{3 l_{1}+2 l_{2}} A^{l_{1}+l_{2}}\left(l_{1}+l_{2}\right) !
$$

for $l_{1}, l_{2}=0,1,2, \ldots$ In a crucial step for obtaining the above derivative estimates is to treat the nonlocal operator $\mathcal{H}_{x}$ which is an essential difference from the KdV equation or nonlinear Schrödinger equations. It is well known that the commutator estimate holds between the Hilbert transform and some smooth cut-off function $a(x)$ (cf. Calderón [3]). However it is now required to show an explicit dependence of the order of the iteration on the constant appeared in the commutator estimate:

$$
\left\|\left[\mathcal{H}_{x}, a^{k}\right] \partial_{x}^{k}\right\|_{\mathcal{L}\left(L^{2} \rightarrow L^{2}\right)} \leq C_{k},
$$

where $a^{k}=a(x)^{k}$. In order to make it explicit, we choose a particular weight function $a(x)=\langle x\rangle^{-2}$, where $\langle x\rangle=\left(1+|x|^{2}\right)^{1 / 2}$ and derive an explicit commuting estimate with the Hilbert transform and $a^{k}$. By this step, we may use the equation (2.4) to gain the regularity and to show the analyticity (2.5).

\section{$\S 3 . \quad$ Preliminary Lemmas}

We give some lemmas for the calculus of the commuting involving the Hilbert transform $\mathcal{H}_{x}$ and weight function $\langle x\rangle^{\delta}$.

First one is well known commuting estimate.

Lemma 3.1. For $p, q \in(1, \infty), r \in(1, \infty]$ and $0<\alpha<1$, we have for $1 / p=1 / r+1 / q$ with

$$
\left\|\left[D_{x}^{\alpha}, f\right] \psi\right\|_{p} \leq C\left\|D_{x}^{\alpha} f\right\|_{r}\|\psi\|_{q}, \quad f \in \dot{H}^{\rho}, \quad g \in L^{r} .
$$

See for example T. Kato and Ponce [32] and Kenig-Ponce-Vega [33]. For some commuting estimate for the fractional derivative, we need the followings (cf. Yosihara [54]). 
Lemma 3.2. We have the following inequalities $\alpha \in(0,1), \rho>(1 / 2)+$ $\alpha$ and

$$
\begin{aligned}
& \left\|\left[D_{x}^{\alpha}, f\right] \psi\right\|_{2} \leq C\|f\|_{W^{1, \infty}}\|\psi\|_{2}, \\
& \left\|\left[D_{x}^{\alpha}, f\right] \psi\right\|_{2} \leq C\|f\|_{H^{\rho}}\|\psi\|_{2}, \\
& \left\|\left[D_{x}^{\alpha},\langle x\rangle^{\beta}\right] \psi\right\|_{2}+\left\|\left[D_{x}^{\alpha} \mathcal{H}_{x},\langle x\rangle^{\beta}\right] \psi\right\|_{2} \leq C\left\|\langle x\rangle^{\beta} \psi\right\|_{2},
\end{aligned}
$$

where $\alpha \in(0,1),|\beta|<\alpha+(1 / 2)$, provided that the right-hand sides are bounded.

Proof of Lemma 3.2. (3.1) and (3.2) are direct results from Lemma 3.1.

We show the first part of (3.3). Noting $\langle x\rangle^{\beta} \leq\langle x+z\rangle^{\beta}+\langle z\rangle^{\beta}$, it follows if $\beta \geq 0$

$$
\begin{aligned}
\left\|\left[D_{x}^{\alpha},\langle x\rangle^{\beta}\right] \psi\right\|_{2}= & \left\|\int \psi(x+z)\left(\langle x+z\rangle^{\beta}-\langle x\rangle^{\beta}\right)|z|^{-1-\alpha} d z\right\|_{2} \\
\leq & C\left\|\int_{|z| \leq 1} \psi(x+z)\langle x+z\rangle^{\beta-1}|z|^{-\alpha} d z\right\|_{2} \\
& +C\left\|\int_{|z|>1} \psi(x+z)|z|^{\beta-1-\alpha} d z\right\|_{2} \\
& +C\left\|\int_{|z|>1} \psi(x+z)\langle x+z\rangle^{\beta}|z|^{-1-\alpha} d z\right\|_{2} \\
\leq & C\left\|\langle x\rangle^{\beta} \psi\right\|_{2}
\end{aligned}
$$

since by the Young inequality $\|f * g\|_{p} \leq\|f\|_{q}\|g\|_{r}$, where $1 \leq p, q, r \leq \infty$ are such that $(1 / r)+(1 / q)=1+(1 / p)$ and by the Hölder inequality $\|f g\|_{p} \leq$ $\|f\|_{q}\|g\|_{r}$, where $1 \leq p, q, r \leq \infty$ are such that $(1 / r)+(1 / q)=1 / p$, we have

$$
\begin{aligned}
\left\|\int_{|z|>1} \psi(x+z)|z|^{\beta-1-\alpha} d z\right\|_{2} & \leq C\|\psi\|_{q}\left(\int_{|z|>1}|z|^{-r(1+\alpha-\beta)} d z\right)^{\frac{1}{r}} \\
& \leq C\left\|\langle x\rangle^{-\beta}\right\|_{p}\left\|\langle x\rangle^{\beta} \psi\right\|_{2} \leq C\left\|\langle x\rangle^{\beta} \psi\right\|_{2},
\end{aligned}
$$

where $q, r \in[1,2], p \in[2, \infty]$ are such that $(1 / q)+(1 / r)=(3 / 2),(1 / p)+$ $(1 / 2)=(1 / q), \beta p>1$ and $r(1+\alpha-\beta)>1$. Such values of $p, q, r$ exist if $0<\beta<(1 / 2)+\alpha$.

For the case $\beta<0$ in view of the inequality

$$
\langle x\rangle^{-|\beta|}\langle z\rangle^{-|\beta|} \leq C\langle x+z\rangle^{-|\beta|}\left(\langle z\rangle^{-|\beta|}+\langle x\rangle^{-|\beta|}\right)
$$


we get

$$
\begin{aligned}
& \left\|\left[D_{x}^{\alpha},\langle x\rangle^{-|\beta|}\right] \psi\right\|_{2} \\
& \leq C\left\|\int_{|z| \leq 1} \psi(x+z)\langle x+z\rangle^{-|\beta|}|z|^{-\alpha} d z\right\|_{2} \\
& +C\left\|\int_{|z|>1} \psi(x+z)\langle x+z\rangle^{-|\beta|}|z|^{-1-\alpha} d z\right\|_{2} \\
& +C\left\|\langle x\rangle^{-|\beta|} \int_{|z|>1} \psi(x+z)\langle x+z\rangle^{-|\beta|}|z|^{|\beta|-1-\alpha} d z\right\|_{2} \\
& \leq C\left\|\langle x\rangle^{-|\beta|} \psi\right\|_{2},
\end{aligned}
$$

where applying the Young and Hölder inequalities we estimated with $p \in$ $[1, \infty]:(1 / p)>(1 / 2)-|\beta|$, and $q \in[1,2]$ such that $1 / q=(1 / 2)+(1 / p)>1-|\beta|$ and $q(1+\alpha-|\beta|)>1$

$$
\begin{aligned}
& \left\|\langle x\rangle^{-|\beta|} \int_{|z|>1} \psi(x+z)\langle x+z\rangle^{-|\beta|}|z|^{|\beta|-1-\alpha} d z\right\|_{2} \\
& \quad \leq C\left\|\int_{|z|>1} \psi(x+z)\langle x+z\rangle^{-|\beta|}|z|^{|\beta|-1-\alpha} d z\right\|_{p} \\
& \quad \leq C\left\|\langle x\rangle^{-|\beta|} \psi\right\|_{2}\left(\int_{|z|>1}|z|^{-q(1+\alpha-|\beta|)} d z\right)^{\frac{1}{q}} \leq C\left\|\langle x\rangle^{-|\beta|} \psi\right\|_{2},
\end{aligned}
$$

since $|\beta|<(1 / 2)+\alpha$. The estimate for $\left\|\left[D_{x}^{\alpha} \mathcal{H}_{x},\langle x\rangle^{\beta}\right] \psi\right\|_{2}$ is considered in the same manner. Lemma 3.2 is proved.

We often use the commuting regularization as follows (see for example Yosihara [54]);

Lemma 3.3. Let $\alpha \in(0,1)$ and $f$ is a smooth function with $|\xi| \hat{f}(\xi)$ being in $L^{1}$. Then we have

$$
\left\|\left[D_{x}^{\alpha}, f\right] \psi\right\|_{2} \leq C\||\xi| \hat{f}\|_{1}\left\|D_{x}^{\alpha-1} \psi\right\|_{2} \quad \text { for } \quad \psi \in \dot{H}^{\alpha-1}
$$

$$
\left\|D_{x}^{1-\alpha}\left[D_{x}^{\alpha}, f\right] \psi\right\|_{2} \leq C\left(\||\xi| \hat{f}\|_{1}\right)\|\psi\|_{2} \quad \text { for } \quad \psi \in L^{2} .
$$

If $\langle\xi\rangle \hat{f}(\xi) \in L^{1}$,

$$
\left\|\left[D_{x}^{\alpha}, f\right] \psi\right\|_{2} \leq C\|\langle\xi\rangle \hat{f}\|_{1}\left\|\left\langle D_{x}\right\rangle^{\alpha-1} \psi\right\|_{2} .
$$


Proof of Lemma 3.3. Those estimates are modified version of the lemma due to [54]. We see that

$$
\mathcal{F}\left(\left[D_{x}^{\alpha}, f\right] \psi\right)=\int_{\mathbb{R}}\left(|\xi|^{\alpha}-|\eta|^{\alpha}\right)|\eta|^{1-\alpha} \hat{f}(\xi-\eta)|\eta|^{\alpha-1} \hat{\psi}(\eta) d \eta
$$

When $|\eta| \leq 1$,

$$
\left.|| \xi\right|^{\alpha}-\left.|\eta|^{\alpha}|| \eta\right|^{1-\alpha} \leq C|\xi-\eta| .
$$

When $|\eta| \geq 1$ and $|\xi| \geq|\eta|$,

$$
\begin{aligned}
\|\left.\xi\right|^{\alpha}-\left.|\eta|^{\alpha}|| \eta\right|^{1-\alpha} & \leq|\xi|^{\alpha}\left(1-\frac{|\eta|^{\alpha}}{|\xi|^{\alpha}}\right)|\eta|^{1-\alpha} \\
& \leq(|\xi|-|\eta|)\left(\frac{|\eta|}{|\xi|}\right)^{1-\alpha} \leq|\xi-\eta|
\end{aligned}
$$

and the other case is similar to obtain

$$
\left.|| \xi\right|^{\alpha}-\left.|\eta|^{\alpha}|| \eta\right|^{1-\alpha} \leq C|\xi-\eta| .
$$

Hence the Hausdorff-Young inequality gives

$$
\left\|\mathcal{F}\left(\left[D_{x}^{\alpha}, f\right] \psi\right)\right\|_{2} \leq\||\xi| \hat{f}\|_{1}\left\|D_{x}^{\alpha-1} \psi\right\|_{2} .
$$

This proves (3.4). The second estimate (3.5) is the dual of (3.4). One can show (3.6) by a simple modification.

Immediately from Lemma 3.3, we particularly have

Corollary 3.4. If $f$ is sufficiently smooth and $\left|\partial_{x} f\right| \leq C\langle x\rangle^{-1 / 2-\varepsilon}$. Then for $0<\alpha \leq 1$ and $\psi \in H^{\alpha-1}$ we have

$$
\left\|\left[D_{x}^{\alpha}, f\right] \psi\right\|_{2} \leq C\left\|D^{\alpha-1} \psi\right\|_{2} .
$$

Lemma 3.5. Let $s \in(0,2)$, we have

$$
\left\|\langle x\rangle^{\nu} f\right\|_{H^{s-\nu}} \leq C\|f\|_{\dot{H}^{s}}+C\left\|\langle x\rangle^{s} f\right\|_{2}
$$

for all $\nu \in(0,(s / 2)+(1 / 4))$ provided that the right-hand side is bounded.

Proof. From Lemma 3.2 we get

$$
\begin{aligned}
\left\|\langle x\rangle^{\nu} D_{x}^{s-\nu} f\right\|_{2}^{2} \leq & \left|\left(\langle x\rangle^{\nu} D_{x}^{s-\nu} f,\left[D_{x}^{\mu},\langle x\rangle^{\nu}\right] D_{x}^{s-\nu-\mu} f\right)\right| \\
& +\left|\left(\left[D_{x}^{\mu},\langle x\rangle^{\nu}\right] D_{x}^{s-\nu} u,\langle x\rangle^{\nu} D_{x}^{s-\nu-\mu} f\right)\right|
\end{aligned}
$$




$$
\begin{aligned}
& +\left|\left(\langle x\rangle^{\nu-\mu} D_{x}^{s-\nu+\mu} f,\langle x\rangle^{\nu+\mu} D_{x}^{s-\nu-\mu} f\right)\right| \\
\leq & C\left\|\langle x\rangle^{\nu-\mu} D_{x}^{s-\nu+\mu} f\right\|_{2}\left\|\langle x\rangle^{\nu+\mu} D_{x}^{s-\nu-\mu} f\right\|_{2} \\
& +C\left\|\langle x\rangle^{\nu} D_{x}^{s-\nu} f\right\|_{2}\left\|\langle x\rangle^{\nu} D_{x}^{s-\nu-\mu} f\right\|_{2}
\end{aligned}
$$

with any $\nu<\mu+(1 / 2), \mu \in(0,1)$, whence

$$
\left\|\langle x\rangle^{\nu} D_{x}^{s-\nu} f\right\|_{2} \leq C\left\|\langle x\rangle^{\nu-\mu} D_{x}^{s-\nu+\mu} f\right\|_{2}+C\left\|\langle x\rangle^{\nu+\mu} D_{x}^{s-\nu-\mu} f\right\|_{2} .
$$

Therefore taking $\mu=\nu$ we obtain

$$
\left\|\langle x\rangle^{\nu} D_{x}^{s-\nu} f\right\|_{2} \leq C\|f\|_{\dot{H}^{s}}+C\left\|\langle x\rangle^{2 \nu} D_{x}^{s-2 \nu} f\right\|_{2}
$$

with $\nu=2^{k} \rho$ for all $0 \leq k \leq n-1$. Since $2^{n} \rho=s$, substituting successively estimate (3.8) with $k$ from $n-1$ to 0 we obtain

$$
\left\|\langle x\rangle^{\nu} D_{x}^{s-\nu} f\right\|_{2} \leq C\|f\|_{\dot{H}^{s}}+C\left\|\langle x\rangle^{s} f\right\|_{2}
$$

with $\nu=2^{k} \rho$ for all $k=0, \ldots, n$.

Now by applying the analytic interpolation theorem, we conclude the desired estimate for any $\nu$.

\section{$\S 4$. Construction of the Solution}

In this section, we show the time local wellposedness for the following system of Benjamin-Ono type equations:

$$
\left\{\begin{array}{l}
\partial_{t} u_{k}+\mathcal{H}_{x} \partial_{x}^{2} u_{k}=B_{k}(u, u), \quad t, x \in \mathbb{R} \\
u_{k}(0, x)=\phi_{k}(x)
\end{array}\right.
$$

where

$$
B_{k}(u, u)=-\partial_{x} \sum_{k=k_{0}+k_{1}+k_{2}} \frac{k !}{k_{0} ! k_{1} ! k_{2} !} u_{k_{1}} u_{k_{2}} .
$$

The space where we solve the system (2.3) is infinite sum of the Sobolev spaces. Let $f=\left(f_{0}, f_{1}, \ldots, f_{k}, \ldots\right)$ denotes the infinite series of distributions and define

$$
\begin{array}{r}
\mathcal{A}_{A_{1}}\left(H^{s} \cap L_{s}^{2}\right)=\left\{f=\left(f_{0}, f_{1}, \ldots, f_{k}, \ldots\right), f_{i} \in C\left([0, T] ; H^{s} \cap L_{s}^{2}\right)\right. \\
\left.(i=0,1,2, \ldots) \quad \text { such that }\|f\|_{\mathcal{A}_{A_{1}}}<\infty\right\}
\end{array}
$$


where

$$
\|f\|_{\mathcal{A}_{A_{1}}} \equiv\left(\sum_{k=0}^{\infty} \frac{A_{1}^{k}}{(k-1) !^{2}}\left\|f_{k}\right\|_{C\left([0, T] ; H^{s} \cap L_{s}^{2}\right)}^{2}\right)^{1 / 2}
$$

with

$$
\|f\|_{H^{s} \cap L_{s}^{2}} \equiv\|f\|_{H^{s}}+\left\|\langle x\rangle^{s} f\right\|_{2} .
$$

Proposition 4.1. Let $s>3 / 2$. Suppose that for some $A_{0}>0$, the initial data $\phi \in H^{s}(\mathbb{R}) \cap L_{s}^{2}(\mathbb{R})$ and satisfies

$$
\sum_{k=0}^{\infty} \frac{A_{0}^{k}}{(k-1) !^{2}}\left\|\phi_{k}\right\|_{H^{s} \cap L_{s}^{2}}^{2}<\infty
$$

then there exists $T>0$ and $A_{1}>0$ such that the system of equations (4.1) with (4.2) is well-posed in the class;

$$
\sum_{k=0}^{\infty} \frac{A_{1}^{k}}{(k-1) !^{2}}\left\|u_{k}\right\|_{C\left([0, T] ; H^{s} \cap L_{s}^{2}\right)}^{2}<\infty .
$$

Remark 4. For the nonlinear Schrödinger equation, the same conclusion holds for $s \geq 0$. For the Korteweg-de Vries equation and nonlinear Schrödinger equations with some exotic nonlinear terms, the similar proposition in the negative exponent Sobolev spaces is proved ([25] and [26]).

By setting $v_{k}=((k-1) !)^{-1} u_{k}$, the equation (4.1)-(4.2) can be reduced as the following slightly simpler system;

$$
\left\{\begin{array}{l}
\partial_{t} v_{k}+\mathcal{H}_{x} \partial_{x}^{2} v_{k}=\tilde{B}_{k}(v, v), \quad t, x \in \mathbb{R} \\
u_{k}(0, x)=\phi_{k}(x)
\end{array}\right.
$$

where

$$
\tilde{B}_{k}(v, v)=-\partial_{x} \sum_{k=k_{0}+k_{1}+k_{2}} \frac{k}{k_{0} ! k_{1} k_{2}} v_{k_{1}} v_{k_{2}} .
$$

Recall that inside the above summation, $k$ is understood as $k ! /(k-1)$ ! so that it is 1 if $k=0$. In the following, we show Proposition 4.1 by dividing into two steps.

To recover regularity loss, we introduce a weight function of exponential type. This argument is originally due to Mizohata [39], Kato [30] and Doi [9], later on it is developed by Chihara [4], Hayashi [13] and Kenig-Ponce-Vega [37]. 
Definition. For $\sigma>0$ and $b>0$, we let

$$
\begin{aligned}
\omega(x) & \equiv b\langle x\rangle^{-1 / 2-\sigma}, \\
E_{x}(x) & \equiv \exp \left(-\int_{-\infty}^{x} \omega^{2}(y) d y\right) .
\end{aligned}
$$

From the definition we see $\left\|E_{x} \psi\right\| \leq\|\psi\|$ and also the inverse operator $E_{x}^{-1}$ is continuous $\left\|E_{x}^{-1} \psi\right\| \leq e^{C b^{2}}\|\psi\|$. In the next lemma we derive an energy estimate, involving the operator $E_{x}$ expressing the smoothing property of the Benjamin-Ono equation originally due to T. Kato [30]. See for some variants in Ginibre-Tsutsumi [10], Ponce [43], Doi [9], Chihara [4] and Hayashi [13].

Lemma 4.2. For a smooth solution u to the linear Benjamin-Ono type equation;

$$
\left\{\begin{array}{l}
\partial_{t} u+\mathcal{H}_{x} \partial_{x}^{2} u=f, \quad t, x \in \mathbb{R} \\
u(0, x)=\phi(x)
\end{array}\right.
$$

the following inequality holds

$$
\begin{aligned}
& \frac{d}{d t}\left\|E_{x} u\right\|_{2}^{2}+2\left\|\omega E_{x} D_{x}^{1 / 2} u\right\|_{2}^{2}+2\left\|\omega E_{x} \mathcal{H}_{x} D_{x}^{1 / 2} u\right\|_{2}^{2} \\
& \quad \leq 2\left|\left(E_{x} u, E_{x} f\right)\right|+C\|u\|_{2}^{2} .
\end{aligned}
$$

Proof of Lemma 4.2. Applying operator $E_{x}$ to both sides of the linear Benjamin-Ono equation (4.6),

$$
\partial_{t} E_{x} u+\mathcal{H}_{x} \partial_{x}^{2} E_{x} u=\left[\mathcal{H}_{x} \partial_{x}^{2}, E_{x}\right] u+E_{x} f .
$$

Since $\left[\partial_{x}, E_{x}\right]=-\omega^{2} E_{x}$, we obtain

$$
\begin{aligned}
\partial_{t} E_{x} u+\mathcal{H}_{x} \partial_{x}^{2} E_{x} u & +2 \omega^{2} E_{x} D_{x} u \\
& =\left(\omega^{4}-\partial_{x} \omega^{2}\right) E_{x} \mathcal{H}_{x} u+\partial_{x}^{2}\left[\mathcal{H}_{x}, E_{x}\right] u+E_{x} f .
\end{aligned}
$$

Therefore multiplying both sides of equation (4.7) by $E_{x} u$ and integrating over $\mathbb{R}$, we have

$$
\begin{aligned}
& \frac{d}{d t}\left\|E_{x} u\right\|_{2}^{2}+4\left(\omega^{2} E_{x} D_{x} u, E_{x} u\right) \\
& \leq 2\left|\left(\left(\omega^{4}-\partial_{x} \omega^{2}\right) E_{x} \mathcal{H}_{x} u, E_{x} u\right)\right|+2\left|\left(\partial_{x}^{2}\left[\mathcal{H}_{x}, E_{x}\right] u, E_{x} u\right)\right|+2\left|\left(E_{x} f, E_{x} u\right)\right| .
\end{aligned}
$$

For the right hand side, it holds $\left\|\left(\omega^{4}-\partial_{x} \omega^{2}\right)\right\|_{\infty} \leq C$ and we have

$$
\left\|\left(\omega^{4}-\partial_{x} \omega^{2}\right) E_{x} \mathcal{H}_{x} u\right\|_{2} \leq C\|u\|_{2} .
$$


By the identity

$$
\frac{d^{2}}{d x^{2}}\left(\frac{f(x)-f(y)}{x-y}\right)=(y-x)^{-3} \int_{x}^{y}(y-\xi)^{2} f^{\prime \prime \prime}(\xi) d \xi
$$

we see that

$$
\begin{aligned}
& \left|\partial_{x}^{2}\left(\frac{e^{-\int_{-\infty}^{y} \omega^{2} d z}-e^{-\int_{-\infty}^{x} \omega^{2} d z}}{y-x}\right)\right| \\
& \quad \leq C\left(\langle x\rangle^{-1}\langle x-y\rangle^{-1}+\langle x\rangle^{-1}\langle y\rangle^{-1}+\langle x-y\rangle^{-1}\langle y\rangle^{-1}\right)
\end{aligned}
$$

whence

$$
\begin{aligned}
\left\|\partial_{x}^{2}\left[\mathcal{H}_{x}, E_{x}\right] u\right\|_{2} & =C\left\|\partial_{x}^{2} \int_{\mathbb{R}} \frac{\exp \left(-\int_{-\infty}^{y} \omega^{2} d z\right)-\exp \left(-\int_{-\infty}^{x} \omega^{2} d z\right)}{y-x} u(y) d y\right\|_{2} \\
& \leq C\|u\|_{2} .
\end{aligned}
$$

Hence it follows that

$$
\frac{d}{d t}\left\|E_{x} u\right\|_{2}^{2}+4\left(\omega^{2} E_{x} D_{x} u, E_{x} u\right) \leq 2\left|\left(E_{x} f, E_{x} u\right)\right|+C\|u\|_{2}^{2} .
$$

From Lemma 3.2, we find

$$
\begin{aligned}
4\left(\omega^{2} E_{x} D_{x} u, E_{x} u\right)= & 4\left(D_{x} \omega E_{x} u, \omega E_{x} u\right)-4\left(\left[D_{x}, \omega E_{x}\right] u, \omega E_{x} u\right) \\
\geq & 2\left\|D_{x}^{1 / 2} \omega E_{x} u\right\|_{2}^{2}+2\left\|\mathcal{H}_{x} D_{x}^{1 / 2} \omega E_{x} u\right\|_{2}^{2}-C\|u\|_{2}^{2} \\
\geq & 2\left\|\omega E_{x} D_{x}^{1 / 2} u\right\|_{2}^{2}+2\left\|\omega E_{x} \mathcal{H}_{x} D_{x}^{1 / 2} u\right\|_{2}^{2} \\
& \quad-\left\|\left[D_{x}^{1 / 2}, \omega E_{x}\right] u\right\|_{2}^{2}-\left\|\left[\mathcal{H}_{x} D_{x}^{1 / 2}, \omega E_{x}\right] u\right\|_{2}^{2}-C\|u\|_{2}^{2} \\
\geq & 2\left\|\omega E_{x} D_{x}^{1 / 2} u\right\|_{2}^{2}+2\left\|\omega E_{x} \mathcal{H}_{x} D_{x}^{1 / 2} u\right\|_{2}^{2}-C\|u\|_{2}^{2} .
\end{aligned}
$$

This proves Lemma 4.2.

We give the weighted nonlinear estimate as follows.

Lemma 4.3. For $0<\sigma<1 / 6$, let $s=(3 / 2)+3 \sigma, \delta=(1 / 2)+\sigma$ and $\omega(x)=b\langle x\rangle^{-\delta}$ for some $b>0$. For $u$, $v$ and $w \in H^{s} \cap L_{s}^{2}$, then we have

$$
\begin{aligned}
& \mid\left(E_{x} u\right.\left.E_{x} D_{x}^{s} \partial_{x}(v w)\right) \mid \\
& \leq C b^{-2}\|w\|_{H^{s} \cap L_{s}^{2}}\left(\left\|\omega E_{x} D_{x}^{s+\frac{1}{2}} \mathcal{H}_{x} v\right\|_{2}+\left\|\omega E_{x} D_{x}^{s+\frac{1}{2}} v\right\|_{2}\right)\left\|\omega E_{x} D_{x}^{\frac{1}{2}} u\right\|_{2} \\
& \quad+C b^{-2}\|v\|_{H^{s} \cap L_{s}^{2}}\left(\left\|\omega E_{x} D_{x}^{s+\frac{1}{2}} \mathcal{H}_{x} w\right\|_{2}+\left\|\omega E_{x} D_{x}^{s+\frac{1}{2}} w\right\|_{2}\right)\left\|\omega E_{x} D_{x}^{\frac{1}{2}} u\right\|_{2} \\
& \quad+C b^{-1}\|v\|_{H^{s} \cap L_{s}^{2}}\|w\|_{H^{s} \cap L_{s}^{2}}\left\|\omega E_{x} D_{x}^{\frac{1}{2}} u\right\|_{2}+C\|u\|_{2}\|v\|_{H^{s} \cap L_{s}^{2}}\|w\|_{H^{s} \cap L_{s}^{2}}
\end{aligned}
$$


Proof of Lemma 4.3. Since $E_{x}$ is smooth and $\left|\partial_{x} E_{x}\right| \in L^{2}$ we have from (3.4) in Lemma 3.3 (or Corollary 3.4) that

(4.10) $\left|\left(E_{x} u, E_{x} D_{x}^{s} \partial_{x}(v w)\right)\right|$

$$
\begin{aligned}
& \leq\left|\left(E_{x} D_{x}^{1 / 2} u, E_{x} D_{x}^{s-\frac{1}{2}} \partial_{x}(v w)\right)\right|+\left|\left(\left[D_{x}^{1 / 2}, E_{x}^{2}\right] u, D_{x}^{s-\frac{1}{2}} \partial_{x}(v w)\right)\right| \\
& \leq C\left\|\langle x\rangle^{-\delta} E_{x} D_{x}^{1 / 2} u\right\|_{2}\left\|\langle x\rangle^{\delta} E_{x} D_{x}^{s-\frac{1}{2}} \partial_{x}(v w)\right\|_{2}+C\|u\|_{2}\left\|\partial_{x}(v w)\right\|_{H^{s-1}} .
\end{aligned}
$$

Noting $s=3 / 2+3 \sigma, \omega=b\langle x\rangle^{-\delta}$, we estimate now by Lemma 3.2

$$
\begin{aligned}
\left\|\langle x\rangle^{\delta} E_{x} D_{x}^{s-\frac{1}{2}} w \partial_{x} v\right\|_{2} \\
=\left\|\langle x\rangle^{\delta} E_{x} D_{x}^{3 \sigma} \mathcal{H}_{x} \partial_{x}\left(w \partial_{x} v\right)\right\|_{2} \\
\leq \| \\
\leq D_{x}^{3 \sigma} \mathcal{H}_{x}\langle x\rangle^{\delta} E_{x} \partial_{x}\left(w \partial_{x} v\right)\left\|_{2}+\right\|\left[D_{x}^{3 \sigma} \mathcal{H}_{x},\langle x\rangle^{\delta} E_{x}\right] \partial_{x}\left(w \partial_{x} v\right) \|_{2} \\
\leq\left\|D_{x}^{3 \sigma} \mathcal{H}_{x}\langle x\rangle^{\delta} E_{x} w \partial_{x}^{2} v\right\|_{2}+\left\|D_{x}^{3 \sigma} \mathcal{H}_{x}\left(\langle x\rangle^{\delta} E_{x} \partial_{x} w \cdot \partial_{x} v\right)\right\|_{2} \\
\quad+\left\|D_{x}^{3 \sigma}\left[\mathcal{H}_{x},\langle x\rangle^{\delta} E_{x}\right] \partial_{x}\left(w \partial_{x} v\right)\right\|_{2}+\left\|\left[D_{x}^{3 \sigma},\langle x\rangle^{\delta} E_{x}\right] \mathcal{H}_{x} \partial_{x}\left(w \partial_{x} v\right)\right\|_{2} \\
\equiv \\
=I_{1}+I_{2}+I_{3}+I_{4} .
\end{aligned}
$$

For the first term in (4.11) we use the Sobolev emmbedding $H^{(1 / 2)+\sigma} \subset L^{\infty}$ and use Lemmas 3.2 and 3.3 again to get

$$
\begin{aligned}
I_{1}= & \left\|D_{x}^{3 \sigma} \mathcal{H}_{x}\langle x\rangle^{1-\sigma} w\langle x\rangle^{-\frac{1}{2}+2 \sigma} E_{x} \partial_{x}^{2} v\right\|_{2} \\
\leq & \left\|\langle x\rangle^{\delta} w D_{x}^{3 \sigma} \mathcal{H}_{x} E_{x} \partial_{x}^{2} v\right\|_{2}+\left\|\langle x\rangle^{1-\sigma} w\left[D_{x}^{3 \sigma} \mathcal{H}_{x},\langle x\rangle^{-\frac{1}{2}+2 \sigma}\right] E_{x} \partial_{x}^{2} v\right\|_{2} \\
& +\left\|\left[D_{x}^{3 \sigma} \mathcal{H}_{x},\langle x\rangle^{1-\sigma} w\right]\langle x\rangle^{-\frac{1}{2}+2 \sigma} E_{x} \partial_{x}^{2} v\right\|_{2} \\
\leq & C\left\|\langle x\rangle^{2 \delta} w\right\|_{\infty}\left(\left\|\langle x\rangle^{-\delta} E_{x} D_{x}^{3 \sigma} \mathcal{H}_{x} \partial_{x}^{2} v\right\|_{2}\right)+C\left\|\langle x\rangle^{1-\sigma} w\right\|_{H^{\frac{1}{2}+4 \sigma}} \\
& \times\left(\left\|\left[D_{x}^{3 \sigma} \mathcal{H}_{x},\langle x\rangle^{-\frac{1}{2}+\sigma}\right] E_{x} \partial_{x}^{2} v\right\|_{2}+\left\|\langle x\rangle^{-\frac{1}{2}+2 \sigma} E_{x} \partial_{x}^{2} v\right\|_{2}\right) \\
\leq & C\left\|\langle x\rangle^{2 \delta} w\right\|_{\infty}\left\|\langle x\rangle^{-\delta} E_{x} \mathcal{H}_{x} D_{x}^{s+\frac{1}{2}} v\right\|_{2} \\
& +C\left\|\langle x\rangle^{1-\sigma} w\right\|_{H^{\frac{1}{2}+4 \sigma}}\left(\left\|\langle x\rangle^{-\frac{1}{2}+2 \sigma} E_{x} \partial_{x}^{2} v\right\|_{2}+\left\|D_{x}^{\frac{1}{2}} \partial_{x} v\right\|_{2}\right) \\
& \left(\text { note that } \sigma<\frac{1}{6}\right) .
\end{aligned}
$$

It follows from Lemma 3.5 that

$$
\left\|\langle x\rangle^{1-\sigma} w\right\|_{H^{\frac{1}{2}+4 \sigma}} \leq C\left\|\langle x\rangle^{s} w\right\|_{2}+C\|w\|_{H^{s}} .
$$

Since

$$
\left\|\langle x\rangle^{-\frac{1}{2}+2 \sigma} \phi\right\|_{H^{2-s}} \leq C\|\phi\|_{2}+C\left\|\langle x\rangle^{-\delta} \phi\right\|_{H^{\frac{1}{2}}},
$$


we set $\phi=E_{x} D_{x}^{s} v$ and obtain by Lemma 3.3

$$
\begin{aligned}
\left\|\langle x\rangle^{-\frac{1}{2}+2 \sigma} E_{x} \partial_{x}^{2} v\right\|_{2} \leq & \left\|D_{x}^{2-s}\langle x\rangle^{-\frac{1}{2}+2 \sigma} E_{x} D_{x}^{s} v\right\|_{2} \\
& +\left\|\left[D_{x}^{2-s},\langle x\rangle^{-\frac{1}{2}+2 \sigma} E_{x}\right] D_{x}^{s} v\right\|_{2} \\
\leq & \left\|D_{x}^{\frac{1}{2}}\langle x\rangle^{-\delta} E_{x} D_{x}^{s} v\right\|_{2}+C\left\|\langle x\rangle^{-\frac{1}{2}+2 \sigma} E_{x}\right\|_{\infty} t\left\|D_{x}^{s} v\right\|_{2} \\
\leq & \left\|\langle x\rangle^{-\delta} E_{x} D_{x}^{\frac{1}{2}+s} v\right\|_{2} \\
& +\left\|\left[D_{x}^{\frac{1}{2}},\langle x\rangle^{-\delta} E_{x}\right] D_{x}^{s} v\right\|_{2}+C\left\|D_{x}^{s} v\right\|_{2} \\
\leq & C\left\|\langle x\rangle^{-\delta} E_{x} D_{x}^{s+\frac{1}{2}} v\right\|_{2}+C\|v\|_{H^{s}} .
\end{aligned}
$$

For the second term of the right hand side (4.11), we employ the commuting estimate and it follows

$$
\begin{aligned}
I_{2}= & \left\|D_{x}^{3 \sigma} \mathcal{H}_{x}\left(\langle x\rangle^{\delta / 2} \partial_{x} w \cdot\langle x\rangle^{\delta / 2} \partial_{x} v\right)\right\|_{2} \\
\leq & \left\|\langle x\rangle^{\delta / 2} \partial_{x} w \cdot D_{x}^{3 \sigma} \mathcal{H}_{x}\langle x\rangle^{\delta / 2} \partial_{x} v\right\|_{2}+\left\|\langle x\rangle^{\delta / 2} \partial_{x} v \cdot D_{x}^{3 \sigma} \mathcal{H}_{x}\langle x\rangle^{\delta / 2} \partial_{x} w\right\|_{2} \\
& +\| D_{x}^{3 \sigma} \mathcal{H}_{x}\left(\langle x\rangle^{\delta / 2} \partial_{x} w \cdot\langle x\rangle^{\delta / 2} \partial_{x} v\right) \\
& \quad-\langle x\rangle^{\delta / 2} \partial_{x} v \cdot D_{x}^{3 \sigma} \mathcal{H}_{x}\langle x\rangle^{\delta / 2} \partial_{x} w-\langle x\rangle^{\delta / 2} \partial_{x} w \cdot D_{x}^{3 \sigma} \mathcal{H}_{x}\langle x\rangle^{\delta / 2} \partial_{x} v \|_{2}
\end{aligned}
$$

Recalling $\delta=(1 / 2)+\sigma$ and $s=(3 / 2)+3 \sigma$, the first two terms in (4.16) can be estimated by Lemma 3.5 that

$$
\begin{aligned}
& \left\|\langle x\rangle^{\delta / 2} \partial_{x} w \cdot D_{x}^{3 \sigma} \mathcal{H}_{x}\langle x\rangle^{\delta / 2} \partial_{x} v\right\|_{2} \\
& \quad \leq\left\|\langle x\rangle^{\delta / 2} \partial_{x} w\right\|_{\frac{4}{1-2 \sigma}}\left\|D_{x}^{3 \sigma} \mathcal{H}_{x}\langle x\rangle^{\delta / 2} \partial_{x} v\right\|_{\frac{4}{1+2 \sigma}} \\
& \quad \leq C\left\|\langle x\rangle^{\frac{1}{4}+\sigma / 2} \partial_{x} w\right\|_{H^{\frac{1}{4}+\frac{\sigma}{2}}}\left\|D_{x}^{3 \sigma}\langle x\rangle^{\frac{1}{4}+\sigma / 2} \partial_{x} v\right\|_{H^{\frac{1}{4}-\frac{\sigma}{2}}} \\
& \quad \leq C\|v\|_{H^{s} \cap L_{s}^{2}}\|w\|_{H^{s} \cap L_{s}^{2}} \\
& \quad\left\|\langle x\rangle^{\delta / 2} \partial_{x} v \cdot D_{x}^{3 \sigma} \mathcal{H}_{x}\langle x\rangle^{\delta / 2} \partial_{x} w\right\|_{2} \\
& \quad \leq C\|v\|_{H^{s} \cap L_{s}^{2}}\|w\|_{H^{s} \cap L_{s}^{2}}
\end{aligned}
$$

For the error term in (4.16), we use the Sobolev inequality and Lemma 3.5 
gives

$$
\begin{aligned}
& \left\|D_{x}^{2 \sigma}\langle x\rangle^{\frac{1}{4}+\sigma / 2} \partial_{x} v\right\|_{4}\left\|D_{x}^{\sigma}\langle x\rangle^{\frac{1}{4}+\sigma} \partial_{x} w\right\|_{4} \\
& \quad \leq C\left\|D_{x}^{2 \sigma}\langle x\rangle^{\frac{1}{4}+\sigma / 2} \partial_{x} v\right\|_{H^{\frac{1}{4}}}\left\|D_{x}^{\sigma}\langle x\rangle^{\frac{1}{4}+\sigma} \partial_{x} w\right\|_{H^{\frac{1}{4}}} \\
& \quad \leq C\|v\|_{H^{s} \cap L_{s}^{2}}\|w\|_{H^{s} \cap L_{s}^{2}}
\end{aligned}
$$

Very much similar estimate appearing in (4.9) implies that

$$
I_{3} \leq C\left\|w \partial_{x} v\right\|_{2} \leq C\|w\|_{H^{s}}\|v\|_{H^{s}}
$$

By noting $\mathcal{F}\left[\partial_{x}\langle x\rangle^{\delta}\right](\xi) \in L_{\xi}^{1}$ (see Appendix for detail) and $\partial_{x} E_{x} \simeq$ $\langle x\rangle^{-1-\sigma} \in H^{1}$, we apply Lemma 3.3 for the last two terms in (4.11) and obtain

$$
\begin{aligned}
I_{4} \leq & \left\|\left[D_{x}^{3 \sigma}, E_{x}\right]\langle x\rangle^{\delta} \mathcal{H}_{x} \partial_{x}\left(w \partial_{x} v\right)\right\|_{2}+\left\|E_{x}\left[D_{x}^{3 \sigma},\langle x\rangle^{\delta}\right] \mathcal{H}_{x} \partial_{x}\left(w \partial_{x} v\right)\right\|_{2} \\
\leq & C\left\|D_{x}^{3 \sigma-1}\langle x\rangle^{\delta} \mathcal{H}_{x} \partial_{x}\left(w \partial_{x} v\right)\right\|_{2}+C\left\|D_{x}^{3 \sigma-1} \mathcal{H}_{x} \partial_{x}\left(w \partial_{x} v\right)\right\|_{2} \\
\leq & C\left\|D_{x}^{3 \sigma}\langle x\rangle^{\delta} \mathcal{H}_{x}\left(w \partial_{x} v\right)\right\|_{2}+C\left\|D_{x}^{3 \sigma-1} x\langle x\rangle^{\delta-2} \mathcal{H}_{x}\left(w \partial_{x} v\right)\right\|_{2} \\
& +C\left\|D_{x}^{3 \sigma}\left(w \partial_{x} v\right)\right\|_{2} \\
\leq & C\left\|D_{x}^{3 \sigma}\langle x\rangle^{\delta} \mathcal{H}_{x}\left(w \partial_{x} v\right)\right\|_{2}+C\left\|w \partial_{x} v\right\|_{2}+C\left\|D_{x}^{3 \sigma}\left(w \partial_{x} v\right)\right\|_{2} \\
\leq & C\|v\|_{H^{s} \cap L_{s}^{2}}\|w\|_{H^{s} \cap L_{s}^{2}}
\end{aligned}
$$

Gathering (4.11) to (4.19), we obtain

$$
\begin{aligned}
\left\|\langle x\rangle^{\delta} E_{x} D_{x}^{s-\frac{1}{2}} w \partial_{x} v\right\|_{2} \leq & C\left\|\langle x\rangle^{2 \delta} w\right\|_{\infty}\left(\left\|\langle x\rangle^{-\delta} E_{x} D_{x}^{2 \sigma} \mathcal{H}_{x} \partial_{x}^{2} v\right\|_{2}+\left\|\langle x\rangle^{-\delta} v\right\|_{2}\right) \\
& +C\left(\|w\|_{L_{s}^{2}}+\|w\|_{H^{s}}\right)\left(\left\|\langle x\rangle^{-\delta} E_{x} D_{x}^{s+\frac{1}{2}} v\right\|_{2}+\|v\|_{H^{s}}\right) \\
& +C\|v\|_{H^{s} \cap L_{s}^{2}}\|w\|_{H^{s} \cap L_{s}^{2}} \\
\leq & C\left\|\langle x\rangle^{2 \delta} w\right\|_{\infty}\left\|\langle x\rangle^{-\delta} E_{x} D_{x}^{s+\frac{1}{2}} \mathcal{H}_{x} v\right\|_{2} \\
& +\left(\|w\|_{H^{s}}+\|w\|_{L_{s}^{2}}\right)\left\|\langle x\rangle^{-\delta} E_{x} D_{x}^{s+\frac{1}{2}} v\right\|_{2} \\
& +C\|v\|_{H^{s} \cap L_{s}^{2}}\|w\|_{H^{s} \cap L_{s}^{2}} .
\end{aligned}
$$

Hence the first term of (4.10) is as follows;

$$
\begin{aligned}
\|\langle x\rangle^{\delta} & E_{x} D_{x}^{s-\frac{1}{2}} \partial_{x}(v w) \|_{2} \\
\leq & C\left(\left\|\langle x\rangle^{2 \delta} w\right\|_{\infty}\left\|\langle x\rangle^{-\delta} E_{x} D_{x}^{s+\frac{1}{2}} \mathcal{H}_{x} v\right\|_{2}\right. \\
& \left.+\left\|\langle x\rangle^{2 \delta} v\right\|_{\infty}\left\|\langle x\rangle^{-\delta} E_{x} D_{x}^{s+\frac{1}{2}} \mathcal{H}_{x} w\right\|_{2}\right) \\
& +\|w\|_{H^{s} \cap L_{s}^{2}}\left\|\langle x\rangle^{-\delta} E_{x} D_{x}^{s+\frac{1}{2}} v\right\|_{2}+\|v\|_{H^{s} \cap L_{s}^{2}}\left\|\langle x\rangle^{-\delta} E_{x} D_{x}^{s+\frac{1}{2}} w\right\|_{2} \\
& +C\|v\|_{H^{s} \cap L_{s}^{2}}\|w\|_{H^{s} \cap L_{s}^{2}} .
\end{aligned}
$$


The last term in (4.10) can handled much similar way. Since $s>(3 / 2)$,

$$
\left\|\partial_{x}(v w)\right\|_{H^{s-1}} \leq\|w\|_{H^{s}}\|v\|_{H^{s}} .
$$

Whence the result of the lemma follows. Lemma 4.3 is proved.

To show Proposition 4.1 holds, we start to solve the first equation of the system, namely the original Benjamin-Ono equations. This result originally due to Saut and Temam [47] for the KdV equations, and then by Saut [46] in the Sobolev space and Iorio Jr. [22] in the weighted Sobolev space.

Proposition 4.4. Let $s>3 / 2$. Suppose that for some $M_{0}>0$, the initial data $\phi \in H^{s}(\mathbb{R}) \cap L_{s}^{2}(\mathbb{R})$ and satisfies

$$
\|\phi\|_{H^{s} \cap L_{s}^{2}}^{2} \leq M_{0},
$$

then there exists $T>0$ such that the Benjamin-Ono equation (1.1) is well-posed in $H^{s}(\mathbb{R}) \cap L_{s}^{2}(\mathbb{R})$ and the solution $u$ satisfies the following estimate

$$
\|u\|_{C\left([0, T] ; H^{s} \cap L_{s}^{2}\right)}^{2}+\int_{0}^{T}\left\|\omega E_{x} \mathcal{H}_{x} D^{s+\frac{1}{2}} u\right\|_{2}^{2} d t+\int_{0}^{T}\left\|\omega E_{x} D^{s+\frac{1}{2}} u\right\|_{2}^{2} d t \leq 4 M_{0} .
$$

The proof of Proposition 4.4 is almost the same in the literature, see for the details [22]. The only part we need to show is the estimate (4.21).

Proof of Proposition 4.4. We apply the operator $D_{x}^{s}$ to the equation (1.1),

$$
\partial_{t} D_{x}^{s} u+\mathcal{H}_{x} \partial_{x}^{2} D_{x}^{s} u=D_{x}^{s} \partial_{x} u^{2}
$$

and from Lemma 4.2 we have the energy type estimate;

$$
\begin{gathered}
\frac{d}{d t}\left\|E_{x} D_{x}^{s} u\right\|_{2}^{2}+2\left\|\omega E_{x} D_{x}^{s+\frac{1}{2}} u\right\|_{2}^{2}+2\left\|\omega E_{x} \mathcal{H}_{x} D_{x}^{s+\frac{1}{2}} u\right\|_{2}^{2} \\
\left.\leq C \mid\left(E_{x} D_{x}^{s} u, E_{x} D_{x}^{s} \partial_{x} u^{2}\right)\right) \mid+C\left\|E_{x} D_{x}^{s} u\right\|_{2}^{2} .
\end{gathered}
$$

Noting that the leading term from the nonlinear term in the right hand side is estimated by the lower order term

$$
\left.\mid\left(E_{x} D_{x}^{s} u, E_{x} D_{x}^{s} \partial_{x} u^{2}\right)\right) \mid \leq C\left(\left\|\partial_{x} u\right\|_{L^{\infty}}+\|u\|_{L^{\infty}}\right)\left\|D_{x}^{s} u\right\|_{2}^{2}+C\|u\|_{H^{s}}^{3} .
$$

Substituting (4.23) into (4.22), 


$$
\begin{aligned}
& \frac{d}{d t}\left\|E_{x} D_{x}^{s} u\right\|_{2}^{2}+2\left(\left\|\omega E_{x} D_{x}^{s+\frac{1}{2}} u\right\|_{2}^{2}+\left\|\omega E_{x} \mathcal{H}_{x} D_{x}^{s+\frac{1}{2}} u\right\|_{2}^{2}\right) \\
& \leq C\|u\|_{H^{s} \cap L_{s}^{2}}^{2}+C\|u\|_{H^{s} \cap L_{s}^{2}}^{3} .
\end{aligned}
$$

By integrating over $[0, T]$, we have

$$
\begin{gathered}
\left\|E_{x} D_{x}^{s} u(t)\right\|_{2}^{2}+2 \int_{0}^{T}\left(\left\|\omega E_{x} D_{x}^{s+\frac{1}{2}} u(t)\right\|_{2}^{2}+\left\|\omega E_{x} \mathcal{H}_{x} D_{x}^{s+\frac{1}{2}} u(t)\right\|_{2}^{2}\right) d t \\
\leq\left\|D_{x}^{s} \phi\right\|_{2}^{2}+C \int_{0}^{T}\left(\|u(t)\|_{H^{s} \cap L_{s}^{2}}^{2}+C\|u(t)\|_{H^{s} \cap L_{s}^{2}}^{3}\right) d t
\end{gathered}
$$

Combining the estimate of the weighted Sobolev space $H^{s}(\mathbb{R}) \cap L_{s}^{2}(\mathbb{R})$,

$$
\begin{gathered}
\|u(t)\|_{H^{s} \cap L_{s}^{2}}^{2}+2 \int_{0}^{T}\left(\left\|\omega E_{x} D_{x}^{s+\frac{1}{2}} u(t)\right\|_{2}^{2}+\left\|\omega E_{x} \mathcal{H}_{x} D_{x}^{s+\frac{1}{2}} u(t)\right\|_{2}^{2}\right) d t \\
\leq\|\phi\|_{H^{s} \cap L_{s}^{2}}^{2}+C \int_{0}^{T}\left(\|u(t)\|_{H^{s} \cap L_{s}^{2}}^{2}+C\|u(t)\|_{H^{s} \cap L_{s}^{2}}^{3}\right) d t .
\end{gathered}
$$

By choosing $T>0$ small if necessary, the Gronwall argument gives the desired estimate (4.21).

To complete the proof of Proposition 4.1, we construct the solution of the system (2.2) with the solution $v_{0} \equiv u$ given by the above proposition which is the crucial part is to establish the local well-posedness.

Proposition 4.5. Let $s>3 / 2$. Suppose that for some $A_{0}>0$, the initial data $\phi \in H^{s}(\mathbb{R}) \cap L_{s}^{2}(\mathbb{R})$ and satisfies

$$
\sum_{k=0}^{\infty} A_{0}^{k}\left\|\phi_{k}\right\|_{H^{s} \cap L_{s}^{2}}^{2}<\infty
$$

then there exists $T>0$ and $A_{1}>0$ such that the system (4.3) with (4.4) is well-posed in the class;

$$
\begin{aligned}
\sum_{k=0}^{\infty} A_{1}^{k}\left(\left\|v_{k}\right\|_{C\left([0, T] ; H^{s} \cap L_{s}^{2}\right)}^{2}\right. & +\int_{0}^{T}\left\|\omega E_{x} \mathcal{H}_{x} D^{s+\frac{1}{2}} v_{k}\right\|_{2}^{2} d t \\
& \left.+\int_{0}^{T}\left\|\omega E_{x} D^{s+\frac{1}{2}} v_{k}\right\|_{2}^{2} d t\right)<\infty .
\end{aligned}
$$

Proof of Proposition 4.5. For a technical reason, we prove the theorem in the case $(3 / 2)<s<(5 / 3)$. The general case is required some modification but 
it is not difficult. Let

$$
M=4\left(M_{0}+\sum_{k=1}^{\infty} A_{0}^{k}\left\|\phi_{k}\right\|_{H^{s} \cap L_{s}^{2}}^{2}\right)^{1 / 2},
$$

where $M_{0}$ is defined as (4.20). For $s \in(3 / 2,5 / 3)$, we set $s=(3 / 2)+3 \sigma$, where $0<\sigma<(1 / 6)$. Let

$$
A_{1}=A_{0} e^{-d M}, \quad d=4 C_{1}\left\|\langle x\rangle^{-1-2 \sigma}\right\|_{1} e^{A_{0}}>0
$$

where $C_{1}$ is an absolute constant determined later. By using the solution $u$ obtained the previous proposition, we define

$$
\begin{aligned}
X_{M}= & \left\{f=\left(f_{0}, f_{1}, f_{2}, \ldots\right) ; f_{j} \in C\left([0, T] ; H^{s} \cap L_{s}^{2}\right), \quad j \in \mathbb{N}, \quad f_{0} \equiv u,\right. \\
\|f\|_{X} \equiv \sum_{k=1}^{\infty} \sup _{t \in[0, T]} A_{1}^{k}\left\|f_{k}(t)\right\|_{H^{s} \cap L_{s}^{2}}^{2} & \\
& \left.+\sum_{k=1}^{\infty} \int_{0}^{T} A_{1}^{k}\left(\left\|\omega E_{x} D_{x}^{s+\frac{1}{2}} f_{k}(t)\right\|_{2}^{2}+\left\|\omega E_{x} \mathcal{H}_{x} D_{x}^{s+\frac{1}{2}} f_{k}(t)\right\|_{2}^{2}\right) d t \leq M^{2}\right\} .
\end{aligned}
$$

Then we introduce a map $\Phi$ on $X_{M}$ as follows; for any $w \in X_{M}, \Phi(w)=$ $v=\left(v_{0}, v_{1}, \ldots\right)$, where $v_{0} \equiv u$ and $v_{k} k \in \mathbb{N}$ solves the linear Benjamin-Ono type equation;

$$
\left\{\begin{array}{l}
\partial_{t} v_{k}+\mathcal{H}_{x} \partial_{x}^{2} v_{k}=\tilde{B}_{k}(w, v), \quad t, x \in \mathbb{R} \\
v_{k}(0, x)=\phi_{k}(x)
\end{array}\right.
$$

with

$$
\tilde{B}_{k}(w, v)=-\partial_{x} \sum_{k=k_{0}+k_{1}+k_{2}} \frac{k}{k_{0} ! k_{1} k_{2}} v_{k_{1}} w_{k_{2}} .
$$

Then we claim that the map $\Phi$ is contraction from $X_{M}$ into $X_{M}$. To this end, we apply the operator $D_{x}^{s}$ to the equation (4.29),

$$
\partial_{t} D_{x}^{s} v_{k}+\mathcal{H}_{x} \partial_{x}^{2} D_{x}^{s} v_{k}=D_{x}^{s} \tilde{B}_{k}(w, v)
$$

and from Lemma 4.2 we have the energy type estimate;

$$
\begin{aligned}
& \frac{d}{d t}\left\|E_{x} D_{x}^{s} v_{k}\right\|_{2}^{2}+2\left\|\omega E_{x} D_{x}^{s+\frac{1}{2}} v_{k}\right\|_{2}^{2}+2\left\|\omega E_{x} \mathcal{H}_{x} D_{x}^{s+\frac{1}{2}} v_{k}\right\|_{2}^{2} \\
& \quad \leq C\left|\left(E_{x} D_{x}^{s} v_{k}, E_{x} D_{x}^{s} \tilde{B}_{k}(w, v)\right)\right|+C\left\|E_{x} D_{x}^{s} v_{k}\right\|_{2}^{2} .
\end{aligned}
$$


According to Lemma 4.3,

$$
\begin{aligned}
&\left|\left(E_{x} D_{x}^{s} v_{k}, E_{x} D_{x}^{s} \partial_{x}\left(v_{k_{1}} w_{k_{2}}\right)\right)\right| \\
& \leq C b^{-2}\left\|w_{k_{2}}\right\|_{H^{s} \cap L_{s}^{2}}\left(\left\|\omega E_{x} \mathcal{H}_{x} D_{x}^{s+\frac{1}{2}} v_{k_{1}}\right\|_{2}\right. \\
&\left.+\left\|\omega E_{x} D_{x}^{s+\frac{1}{2}} v_{k_{1}}\right\| \|_{2}\right)\left\|\omega E_{x} D_{x}^{s+\frac{1}{2}} v_{k}\right\|_{2} \\
&+C b^{-2}\left\|v_{k_{1}}\right\|_{H^{s} \cap L_{s}^{2}}\left(\left\|\omega E_{x} \mathcal{H}_{x} D_{x}^{s+\frac{1}{2}} w_{k_{2}}\right\|_{2}\right. \\
&\left.+\left\|\omega E_{x} D_{x}^{s+\frac{1}{2}} w_{k_{2}}\right\|_{2}\right)\left\|\omega E_{x} D_{x}^{s+\frac{1}{2}} v_{k}\right\|_{2} \\
&+C b^{-1}\left\|v_{k_{1}}\right\|_{H^{s} \cap L_{s}^{2}}\left\|w_{k_{2}}\right\|_{H^{s} \cap L_{s}^{2}}\left\|\omega E_{x} D_{x}^{s+\frac{1}{2}} v_{k}\right\|_{2} \\
&+C\left\|v_{k}\right\|_{H^{s} \cap L_{s}^{2}}\left\|v_{k_{1}}\right\|_{H^{s} \cap L_{s}^{2}}\left\|w_{k_{2}}\right\|_{H^{s} \cap L_{s}^{2} .}
\end{aligned}
$$

Substituting (4.32) into (4.31),

$$
\begin{aligned}
\frac{d}{d t} \sum_{k=0}^{N} & A_{1}^{k}\left\|E_{x} D_{x}^{s} v_{k}\right\|_{2}^{2}+2 \sum_{k=1}^{N} A_{1}^{k}\left(\left\|\omega E_{x} D_{x}^{s+\frac{1}{2}} v_{k}\right\|_{2}^{2}+\left\|\omega E_{x} \mathcal{H}_{x} D_{x}^{s+\frac{1}{2}} v_{k}\right\|_{2}^{2}\right) \\
\leq & C_{1} b^{-2} \sum_{k=1}^{N} A_{1}^{k}\left\|\omega E_{x} D_{x}^{s+\frac{1}{2}} v_{k}\right\|_{2} \sum_{k=k_{0}+k_{1}+k_{2}} \frac{k}{k_{0} ! k_{1} k_{2}}\left\|w_{k_{2}}\right\|_{H^{s} \cap L_{s}^{2}} \\
& \times\left(\left\|\omega E_{x} D_{x}^{s+\frac{1}{2}} v_{k_{1}}\right\|_{2}+\left\|\omega E_{x} \mathcal{H}_{x} D_{x}^{s+\frac{1}{2}} v_{k_{1}}\right\|_{2}\right) \\
+ & C_{1} b^{-2} \sum_{k=1}^{N} A_{1}^{k}\left\|\omega E_{x} D_{x}^{s+\frac{1}{2}} v_{k}\right\|_{2} \sum_{k=k_{0}+k_{1}+k_{2}} \frac{k}{k_{0} ! k_{1} k_{2}}\left\|v_{k_{1}}\right\|_{H^{s} \cap L_{s}^{2}} \\
& \times\left(\left\|\omega E_{x} D_{x}^{s+\frac{1}{2}} w_{k_{2}}\right\|_{2}+\left\|\omega E_{x} \mathcal{H}_{x} D_{x}^{s+\frac{1}{2}} w_{k_{2}}\right\|_{2}\right) \\
+ & C_{1} b^{-1} \sum_{k=1}^{N} A_{1}^{k} \sum_{k=k_{0}+k_{1}+k_{2}} \\
& \times \frac{k}{k_{0} ! k_{1} k_{2}}\left\|w_{k_{2}}\right\|_{H^{s} \cap L_{s}^{2}}\left\|v_{k_{1}}\right\|_{H^{s} \cap L_{s}^{2}}\left\|\omega E_{x} D_{x}^{s+\frac{1}{2}} v_{k}\right\|_{2} \\
+ & C \sum_{k=1}^{N} A_{1}^{k}\left\|v_{k}\right\|_{H^{s} \cap L_{s}^{2}} \sum_{k=k_{0}+k_{1}+k_{2}} \frac{k}{k_{0} ! k_{1} k_{2}}\left\|v_{k_{1}}\right\|_{H^{s} \cap L_{s}^{2}}\left\|w_{k_{2}}\right\|_{H^{s} \cap L_{s}^{2}} \\
+ & C \sum_{k=1}^{N} A_{1}^{k}\left\|v_{k}\right\|_{H^{s} \cap L_{s}^{2}}^{2}
\end{aligned}
$$

Integrating the result with respect to $t \in[0, T]$ and taking a sum over $k$ 
from 1 to $N$ we find

$$
\begin{aligned}
& \sum_{k=1}^{N} \sup _{t \in[0, T]} A_{1}^{k}\left\|E_{x} D_{x}^{s} v_{k}\right\|_{2}^{2} \\
& +2 \int_{0}^{T} \sum_{k=1}^{N} A_{1}^{k}\left(\left\|\omega E_{x} D_{x}^{s+\frac{1}{2}} v_{k}\right\|_{2}^{2}+\left\|\omega E_{x} \mathcal{H}_{x} D_{x}^{s+\frac{1}{2}} v_{k}\right\|_{2}^{2}\right) d t \\
& \leq \sum_{k=1}^{N} A_{1}^{k}\left\|E_{x} D_{x}^{s} \phi_{k}\right\|_{2}^{2}+C_{1} b^{-2} \int_{0}^{T} \sum_{k=1}^{N} A_{1}^{k}\left\|\omega E_{x} D_{x}^{s+\frac{1}{2}} v_{k}\right\|_{2} \\
& \times \sum_{k=k_{0}+k_{1}+k_{2}} \frac{k}{k_{0} ! k_{1} k_{2}}\left\|w_{k_{2}}\right\|_{H^{s} \cap L_{s}^{2}} \\
& \times\left(\left\|\omega E_{x} D_{x}^{s+\frac{1}{2}} v_{k_{1}}\right\|_{2}+\left\|\omega E_{x} \mathcal{H}_{x} D_{x}^{s+\frac{1}{2}} v_{k_{1}}\right\|_{2}\right) d t \\
& +C_{1} b^{-2} \int_{0}^{T} \sum_{k=1}^{N} A_{1}^{k}\left\|\omega E_{x} D_{x}^{s+\frac{1}{2}} v_{k}\right\|_{2} \\
& \times \sum_{k=k_{0}+k_{1}+k_{2}} \frac{k}{k_{0} ! k_{1} k_{2}}\left\|v_{k_{1}}\right\|_{H^{s} \cap L_{s}^{2}} \\
& \times\left(\left\|\omega E_{x} D_{x}^{s+\frac{1}{2}} w_{k_{2}}\right\|_{2}+\left\|\omega E_{x} \mathcal{H}_{x} D_{x}^{s+\frac{1}{2}} w_{k_{2}}\right\|_{2}\right) d t \\
& +C_{1} b^{-1} \int_{0}^{T} \sum_{k=1}^{N} A_{1}^{k} \\
& \times \sum_{k=k_{0}+k_{1}+k_{2}} \frac{k}{k_{0} ! k_{1} k_{2}}\left\|w_{k_{2}}\right\|_{H^{s} \cap L_{s}^{2}}\left\|v_{k_{1}}\right\|_{H^{s} \cap L_{s}^{2}}\left\|\omega E_{x} D_{x}^{s+\frac{1}{2}} v_{k}\right\|_{2} d t \\
& +C \int_{0}^{T} \sum_{k=1}^{N} A_{1}^{k}\left\|v_{k}\right\|_{H^{s} \cap L_{s}^{2}} \sum_{k=k_{0}+k_{1}+k_{2}} \frac{k}{k_{0} ! k_{1} k_{2}}\left\|v_{k_{1}}\right\|_{H^{s} \cap L_{s}^{2}}\left\|w_{k_{2}}\right\|_{H^{s} \cap L_{s}^{2}} d t \\
& +C \int_{0}^{T} \sum_{k=1}^{N} A_{1}^{k}\left\|v_{k}\right\|_{H^{s} \cap L_{s}^{2}}^{2} d t .
\end{aligned}
$$

Since

$$
\begin{aligned}
& \sum_{k=1}^{\infty} A_{1}^{k}\left\|v_{k}\right\|_{H^{s} \cap L_{s}^{2}} \sum_{k=k_{0}+k_{1}+k_{2}} \frac{k}{k_{0} ! k_{1} k_{2}}\left\|v_{k_{1}}\right\|_{H^{s} \cap L_{s}^{2}}\left\|w_{k_{2}}\right\|_{H^{s} \cap L_{s}^{2}} \\
& \quad \leq\left(\sum_{k=1}^{\infty} A_{1}^{k}\left\|v_{k}\right\|_{H^{s} \cap L_{s}^{2}}^{2}\right)^{1 / 2}
\end{aligned}
$$




$$
\begin{aligned}
& \times\left(\sum_{k=1}^{\infty}\left(\sum_{\tilde{k}=k_{0}+k_{1}+k_{2}} \frac{k^{2}}{k_{0}^{2} k_{1}^{2} k_{2}^{2}}\right)\right. \\
& \left.\times\left(\sum_{\tilde{k}=k_{0}+k_{1}+k_{2}} \frac{A_{1}^{k_{0}}}{\left(k_{0}-1\right) !^{2}} A_{1}^{k_{1}}\left\|v_{k_{1}}\right\|_{H^{s} \cap L_{s}^{2}}^{2} A_{1}^{k_{2}}\left\|w_{k_{2}}\right\|_{H^{s} \cap L_{s}^{2}}^{2}\right)\right)^{1 / 2} \\
\leq & \left(\sum_{k=1}^{\infty} A_{1}^{k}\left\|v_{k}\right\|_{H^{s} \cap L_{s}^{2}}^{2}\right)^{1 / 2} \\
& \times\left(\left(\sup _{k} \sum_{\tilde{k}=k_{0}+k_{1}+k_{2}} \frac{k^{2}}{k_{0}^{2} k_{1}^{2} k_{2}^{2}}\right)\right. \\
& \left.\cdot \sum_{k_{0}=0}^{\infty} \frac{A_{1}^{k_{0}}}{\left(k_{0}-1\right) !^{2}} \sum_{k_{1}=0}^{\infty} A_{1}^{k_{1}}\left\|v_{k_{1}}\right\|_{H^{s} \cap L_{s}^{2}}^{2} \sum_{k_{2}=0}^{\infty} A_{1}^{k_{2}}\left\|w_{k_{2}}\right\|_{H^{s} \cap L_{s}^{2}}^{2}\right)^{1 / 2} \\
\leq & C e^{A_{1}}\left(\sum_{k=1}^{\infty} A_{1}^{k}\left\|v_{k}\right\|_{H^{s} \cap L_{s}^{2}}^{2} \sum_{k=0}^{\infty} A_{1}^{k_{1}}\left\|v_{k}\right\|_{H^{s} \cap L_{s}^{2}}^{2} \sum_{k=0}^{\infty} A_{1}^{k_{2}}\left\|w_{k}\right\|_{H^{s} \cap L_{s}^{2}}^{2}\right)^{1 / 2}
\end{aligned}
$$

(here the meaning of the summation of $\tilde{k}$ is understood as $k_{j}$ is replaced by $k_{j} \vee 1$ ), we see by passing $N \rightarrow \infty$ in (4.34) and the Hölder inequality that

$$
\begin{aligned}
& \sum_{k=1}^{\infty} \sup _{t \in[0, T]} A_{1}^{k}\left\|E_{x} D_{x}^{s} v_{k}\right\|_{2}^{2} \\
& \quad+2 \sum_{k=1}^{\infty} \int_{0}^{T} A_{1}^{k}\left(\left\|\omega E_{x} D_{x}^{s+\frac{1}{2}} v_{k}\right\|_{2}^{2}+\left\|\omega E_{x} \mathcal{H}_{x} D_{x}^{s+\frac{1}{2}} v_{k}\right\|_{2}^{2}\right) d t \\
& \leq \sum_{k=1}^{\infty} A_{1}^{k}\left\|E_{x} D_{x}^{s} \phi_{k}\right\|_{2}^{2}+C_{1} e^{A_{1}} b^{-2}\|w\|_{X}\left(\int_{0}^{T} \sum_{k=1}^{\infty} A_{1}^{k}\left\|\omega E_{x} D_{x}^{s+\frac{1}{2}} v_{k}\right\|_{2}^{2} d t\right)^{1 / 2} \\
& \quad \times\left(\int_{0}^{T} \sum_{k_{1}=0}^{\infty} A_{1}^{k_{1}}\left(\left\|\omega E_{x} D_{x}^{s+\frac{1}{2}} v_{k_{1}}\right\|_{2}^{2}+\left\|\omega E_{x} \mathcal{H}_{x} D_{x}^{s+\frac{1}{2}} v_{k_{1}}\right\|_{2}^{2}\right) d t\right)^{1 / 2} \\
& \quad+C_{1} e^{A_{1}} b^{-2}\|w\|_{X} \int_{0}^{T}\left(\sum_{k=1}^{\infty} A_{1}^{k}\left\|\omega E_{x} D_{x}^{s+\frac{1}{2}} v_{k}\right\|_{2}^{2} \sum_{k_{1}=0}^{\infty} A_{1}^{k_{1}}\left\|v_{k_{1}}\right\|_{H^{s} \cap L_{s}^{2}}^{2}\right)^{1 / 2} d t \\
& \quad+C_{1} b^{-1} e^{A_{1}}\|w\|_{X} \int_{0}^{T}\left(\sum_{k_{1}=0}^{\infty} A_{1}^{k_{1}}\left\|v_{k_{1}}\right\|_{H^{s} \cap L_{s}^{2}}^{2} \sum_{k=1}^{\infty} A_{1}^{k}\left\|\omega E_{x} D_{x}^{s+\frac{1}{2}} v_{k}\right\|_{2}^{2}\right)^{1 / 2} d t
\end{aligned}
$$




$$
\begin{aligned}
& +C_{1}\left(e^{A_{1} / 2}\|w\|_{X}+1\right) \int_{0}^{T} \sum_{k=0}^{\infty} A_{1}^{k}\left\|v_{k}\right\|_{H^{s} \cap L_{s}^{2}}^{2} d t \\
& \leq \sum_{k=1}^{\infty} A_{1}^{k}\left\|E_{x} D_{x}^{s} \phi_{k}\right\|_{2}^{2} \\
& +C_{1} e^{A_{1}} b^{-2} M \int_{0}^{T} \sum_{k_{1}=0}^{\infty} A_{1}^{k}\left(\left\|\omega E_{x} D_{x}^{s+\frac{1}{2}} v_{k}\right\|_{2}^{2}+\left\|\omega E_{x} \mathcal{H}_{x} D_{x}^{s+\frac{1}{2}} v_{k}\right\|_{2}^{2}\right) d t \\
& +C_{1} e^{A_{1}}\left(b^{-2}+b^{-1}\right) M\left(\sum_{k_{1}=0}^{\infty} \sup _{t \in[0, T]} A_{1}^{k_{1}}\left\|v_{k_{1}}\right\|_{H^{s} \cap L_{s}^{2}}^{2}\right)^{1 / 2} \\
& \times T^{1 / 2}\left(\int_{0}^{T} \sum_{k=1}^{\infty} A_{1}^{k}\left\|\omega E_{x} D_{x}^{s+\frac{1}{2}} v_{k}\right\|_{2}^{2} d t\right)^{1 / 2} \\
& +C_{1}\left(e^{A_{1} / 2} M+1\right) \int_{0}^{T} \sum_{k=1}^{\infty} A_{1}^{k}\left\|v_{k}\right\|_{H^{s} \cap L_{s}^{2}}^{2} d t \\
& \leq \sum_{k=1}^{\infty} A_{1}^{k}\left\|E_{x} D_{x}^{s} \phi_{k}\right\|_{2}^{2} \\
& +\left(C_{1} e^{A_{1}} b^{-2} M+\frac{1}{2}\right) \int_{0}^{T} \sum_{k_{1}=1}^{\infty} A_{1}^{k} \\
& \times\left(\left\|\omega E_{x} D_{x}^{s+\frac{1}{2}} v_{k}\right\|_{2}^{2}+\left\|\omega E_{x} \mathcal{H}_{x} D_{x}^{s+\frac{1}{2}} v_{k}\right\|_{2}^{2}\right) d t \\
& +C_{1} e^{A_{1}} b^{-2} M \int_{0}^{T}\left(\left\|\omega E_{x} D_{x}^{s+\frac{1}{2}} v_{0}\right\|_{2}^{2}+\left\|\omega E_{x} \mathcal{H}_{x} D_{x}^{s+\frac{1}{2}} v_{0}\right\|_{2}^{2}\right) d t \\
& +C_{1}^{2} e^{2 A_{0}}\left(b^{-2}+b^{-1}\right)^{2} M^{2} T\left(\sum_{k_{1}=0}^{\infty} \sup _{t \in[0, T]} A_{1}^{k_{1}}\left\|v_{k_{1}}\right\|_{H^{s} \cap L_{s}^{2}}^{2}\right) \\
& +C_{1}\left(e^{A_{0} / 2} M+1\right) \int_{0}^{T} \sum_{k=0}^{\infty} A_{1}^{k}\left\|v_{k}\right\|_{H^{s} \cap L_{s}^{2}}^{2} d t .
\end{aligned}
$$

We now choose sufficiently large $b>0$ in (4.36) such that $C_{1} e^{A_{0}}\left(b^{-2}+\right.$ $\left.b^{-1}\right) M<(1 / 2)$. For simplicity we choose particularly $b^{2}=2 M C_{1} e^{A_{1}}$. Then it follows from (4.36) that

$$
\begin{aligned}
& \sum_{k=1}^{\infty} \sup _{t \in[0, T]} A_{1}^{k}\left\|E_{x} D_{x}^{s} v_{k}\right\|_{2}^{2} \\
& \quad+\int_{0}^{T} \sum_{k=1}^{\infty} A_{1}^{k}\left(\left\|\omega E_{x} D_{x}^{s+\frac{1}{2}} v_{k}\right\|_{2}^{2}+\left\|\omega E_{x} \mathcal{H}_{x} D_{x}^{s+\frac{1}{2}} v_{k}\right\|_{2}^{2}\right) d t
\end{aligned}
$$




$$
\begin{aligned}
\leq & \sum_{k=1}^{\infty} A_{1}^{k}\left\|E_{x} D_{x}^{s} \phi_{k}\right\|_{2}^{2} \\
& +\frac{1}{2} \int_{0}^{T}\left(\left\|\omega E_{x} D_{x}^{s+\frac{1}{2}} v_{0}\right\|_{2}^{2}+\left\|\omega E_{x} \mathcal{H}_{x} D_{x}^{s+\frac{1}{2}} v_{0}\right\|_{2}^{2}\right) d t \\
& +C_{1}\left(e^{A_{1} / 2} M+2\right) T\left(\sum_{k=1}^{\infty} \sup _{t \in[0, T]} A_{1}^{k}\left\|v_{k}\right\|_{H^{s} \cap L_{s}^{2}}^{2}\right) .
\end{aligned}
$$

Since $\left\|D_{x}^{s} v_{k}(t)\right\|_{2} \leq e^{C b^{2}}\left\|E_{x} D_{x}^{s} v_{k}\right\|_{2}$, we have that

$$
\begin{aligned}
\sum_{k=1}^{\infty} \sup _{t \in[0, T]} A_{1}^{k}\left\|D_{x}^{s} v_{k}\right\|_{2}^{2} & \\
& +\int_{0}^{T} \sum_{k=1}^{\infty} A_{1}^{k}\left(\left\|\omega E_{x} D_{x}^{s+\frac{1}{2}} v_{k}\right\|_{2}^{2}+\left\|\omega E_{x} \mathcal{H}_{x} D_{x}^{s+\frac{1}{2}} v_{k}\right\|_{2}^{2}\right) d t \\
\leq & e^{d M} \sum_{k=1}^{\infty} A_{1}^{k}\left\|E_{x} D_{x}^{s} \phi_{k}\right\|_{2}^{2} \\
& +\frac{e^{d M}}{2} \int_{0}^{T}\left(\left\|\omega E_{x} D_{x}^{s+\frac{1}{2}} v_{0}\right\|_{2}^{2}+\left\|\omega E_{x} \mathcal{H}_{x} D_{x}^{s+\frac{1}{2}} v_{0}\right\|_{2}^{2}\right) d t \\
& +C_{1}\left(e^{A_{0} / 2} M+2\right) e^{d M} T\left(\sum_{k_{1}=0}^{\infty} \sup _{t \in[0, T]} A_{1}^{k_{1}}\left\|v_{k_{1}}\right\|_{H^{s} \cap L_{s}^{2}}^{2}\right)
\end{aligned}
$$

where $d=4 C C_{1} e^{A_{0}}$ defined as in (4.27) with $C=\left\|\langle x\rangle^{-1-2 \sigma}\right\|_{1}$. Since

$$
e^{d M} \sum_{k=1}^{\infty} A_{1}^{k}\left\|E_{x} D_{x}^{s} \phi_{k}\right\|_{2}^{2} \leq e^{d M} \frac{A_{1}}{A_{0}} \sum_{k=1}^{\infty} A_{0}^{k}\left\|D_{x}^{s} \phi_{k}\right\|_{2}^{2} \leq \frac{M^{2}}{16}
$$

and $v_{0}=u$ has determined by Proposition 4.4, we can choose $T$ small enough such that

$$
\frac{e^{d M}}{2} \int_{0}^{T}\left(\left\|\omega E_{x} D_{x}^{s+\frac{1}{2}} v_{0}\right\|_{2}^{2}+\left\|\omega E_{x} \mathcal{H}_{x} D_{x}^{s+\frac{1}{2}} v_{0}\right\|_{2}^{2}\right) d t<\frac{M^{2}}{16}
$$

and also

$$
T C_{1}\left(e^{A_{0} / 2} M+2\right) e^{d M}<1 / 2 .
$$


Then it follows from (4.38) and (4.39) that

$$
\begin{aligned}
& \sum_{k=1}^{\infty} \sup _{t \in[0, T]} A_{1}^{k}\left\|D_{x}^{s} v_{k}\right\|_{2}^{2} \\
& \quad+\int_{0}^{T} \sum_{k=1}^{\infty} A_{1}^{k}\left(\left\|\omega E_{x} D_{x}^{s+\frac{1}{2}} v_{k}\right\|_{2}^{2}+\left\|\omega E_{x} \mathcal{H}_{x} D_{x}^{s+\frac{1}{2}} v_{k}\right\|_{2}^{2}\right) d t \\
& \quad \leq \frac{M^{2}}{8}
\end{aligned}
$$

Multiplying the equation (4.29) by $\langle x\rangle^{2 s} v_{k}$ and integrating over $x \in \mathbb{R}$ we obtain

$$
\begin{aligned}
\frac{d}{d t}\left\|\langle x\rangle^{s} v_{k}\right\|_{2}^{2} & +2\left(\langle x\rangle^{s} v_{k},\langle x\rangle^{s} \mathcal{H} \partial_{x}^{2} v_{k}\right) \\
& \leq C \sum_{k=k_{0}+k_{1}+k_{2}} \frac{1}{k_{0} ! k_{1} k_{2}}\left|\left(\langle x\rangle^{s} v_{k},\langle x\rangle^{s} \partial_{x}\left(w_{k_{2}} v_{k_{1}}\right)\right)\right|
\end{aligned}
$$

By identity (4.8) we get

$$
\left|\partial_{x}^{2}\left(\frac{\langle y\rangle^{s}-\langle x\rangle^{s}}{y-x}\right)\right| \leq C\left(\langle x\rangle^{-\delta}\langle x-y\rangle^{-\delta}+\langle x\rangle^{-\delta}\langle y\rangle^{-\delta}+\langle x-y\rangle^{-\delta}\langle y\rangle^{-\delta}\right)
$$

whence

$$
\left\|\partial_{x}^{2}\left[\mathcal{H}_{x},\langle x\rangle^{s}\right] u\right\|_{2}=C \| \partial_{x}^{2} \text { p.v. } \int_{\mathbb{R}} \frac{\langle y\rangle^{s}-\langle x\rangle^{s}}{y-x} u(y) d y\left\|_{2} \leq C\right\| u \|_{2} .
$$

Therefore

$$
\begin{aligned}
\left(\langle x\rangle^{s} v_{k},\langle x\rangle^{s} \mathcal{H} \partial_{x}^{2} v_{k}\right)= & \left(\langle x\rangle^{s} v_{k}, \mathcal{H}_{x} \partial_{x}^{2}\langle x\rangle^{s} v_{k}\right) \\
& +\left(\langle x\rangle^{s} v_{k}, \partial_{x}^{2}\left[\mathcal{H}_{x},\langle x\rangle^{s}\right] v_{k}\right)+C\left(\langle x\rangle^{s} v_{k},\langle x\rangle^{s-2} \mathcal{H}_{x} v_{k}\right),
\end{aligned}
$$

whence by Lemma 3.5 we have

$$
\begin{aligned}
& \left|\left(\langle x\rangle^{s} v_{k},\langle x\rangle^{s} \mathcal{H}_{x} \partial_{x}^{2} v_{k}\right)\right| \\
& \quad \leq C\left\|\langle x\rangle^{s} v_{k}\right\|_{2}\left\|v_{k}\right\|_{2}+C\left\|\langle x\rangle^{s} v_{k}\right\|_{2}\left\|\langle x\rangle^{s-1} \mathcal{H}_{x} \partial_{x} v_{k}\right\|_{2} \\
& \quad \leq C\left\|\langle x\rangle^{s} v_{k}\right\|_{2}\left\|v_{k}\right\|_{H^{s} \cap L_{s}^{2}} .
\end{aligned}
$$


The nonlinear term is estimated similarly as (4.35),

$$
\begin{aligned}
& \sum_{k=1}^{\infty} \sum_{k=k_{0}+k_{1}+k_{2}} \frac{k A_{1}^{k}}{k_{0} ! k_{1} k_{2}}\left|\left(\langle x\rangle^{s} v_{k},\langle x\rangle^{s} \partial_{x}\left(v_{k_{1}} w_{k_{2}}\right)\right)\right| \\
& \quad \leq C e^{A_{1}}\left(\sum_{k=1}^{\infty} A_{1}^{k}\left\|v_{k}\right\|_{L_{s}^{2}}^{2} \sum_{k_{1}=0}^{\infty} A_{1}^{k_{1}}\left\|v_{k_{1}}\right\|_{H^{s} \cap L_{s}^{2}}^{2} \sum_{k_{2}=0}^{\infty} A_{1}^{k_{2}}\left\|w_{k_{2}}\right\|_{H^{s} \cap L_{s}^{2}}^{2}\right)^{1 / 2} \\
& \quad \leq C e^{A_{1}}\|w\| X \sum_{k=1}^{\infty} A_{1}^{k}\left\|v_{k}\right\|_{H^{s} \cap L_{s}^{2}}^{2}
\end{aligned}
$$

Substituting (4.42) into (4.41), integrating with respect to $t \in[0, T]$, multiplying $A_{1}^{k}$ and taking a sum over $k$, we obtain

$$
\begin{aligned}
\sum_{k=1}^{\infty} \sup _{t \in[0, T]} A_{1}^{k}\left\|\langle x\rangle^{s} v_{k}\right\|_{2}^{2} \leq & \frac{M^{2}}{16}+C \sum_{k=1}^{\infty} \int_{0}^{T} A_{1}^{k}\left\|\langle x\rangle^{s} v_{k}\right\|_{2}\left\|v_{k}\right\|_{H^{s} \cap L_{s}^{2}} d t \\
& +C\|w\|_{X} \int_{0}^{T} \sum_{k=1}^{\infty} A_{1}^{k}\left\|v_{k}\right\|_{H^{s} \cap L_{s}^{2}}^{2} d t \\
\leq & \frac{M^{2}}{16}+C(1+M) T \sum_{k=1}^{\infty} \sup _{t \in[0, T]} A_{1}^{k}\left\|v_{k}\right\|_{H^{s} \cap L_{s}^{2}}^{2}
\end{aligned}
$$

Combining the estimates (4.38), (4.44) and (4.21) and choosing sufficiently small time $T>0$, we obtain the estimate

$$
\begin{aligned}
\|v\|_{X}= & \sum_{k=0}^{\infty} \sup _{t \in[0, T]} A_{1}^{k}\left\|v_{k}\right\|_{H^{s} \cap L_{s}^{2}}^{2} \\
& +\sum_{k=1}^{\infty} \int_{0}^{T} A_{1}^{k}\left(\left\|\omega E_{x} D_{x}^{s+\frac{1}{2}} v_{k}\right\|_{2}^{2}+\left\|\omega E_{x} \mathcal{H}_{x} D_{x}^{s+\frac{1}{2}} v_{k}\right\|_{2}^{2}\right) d t \\
\leq & \frac{M^{2}}{4}
\end{aligned}
$$

By virtue of (4.45) we see now that the mapping $\Phi$ is from $X_{M}$ into itself. In the same manner we can prove that for $w, \widetilde{w} \in X_{T}$,

$$
\begin{aligned}
& \sum_{k=1}^{\infty} \sup _{t \in[0, T]} A_{1}^{k}\left\|v_{k}-\widetilde{v}_{k}\right\|_{H^{s} \cap L_{s}^{2}}^{2} \\
& \quad+\int_{0}^{T} \sum_{k=1}^{\infty} A_{1}^{k}\left(\left\|\omega E_{x} D_{x}^{s+\frac{1}{2}}\left(v_{k}-\widetilde{v}_{k}\right)\right\|_{2}^{2}+\left\|\omega E_{x} \mathcal{H}_{x} D_{x}^{s+\frac{1}{2}}\left(v_{k}-\widetilde{v}_{k}\right)\right\|_{2}^{2}\right) d t \\
& \quad<\frac{1}{4}\|w-\widetilde{w}\|_{X}^{2}
\end{aligned}
$$


if $T>0$ is sufficiently small, where $v=\Phi(w)$ and $\widetilde{v}=\Phi(\widetilde{w})$. Thus $\Phi$ is a contraction mapping in $X_{T}$. Therefore there exists a unique solution $u$ of the Cauchy problem (4.29) such that

$$
\sum_{k=1}^{\infty} \sup _{t \in[0, T]} A_{1}^{k}\left\|v_{k}(t)\right\|_{H^{s} \cap L_{s}^{2}}^{2} \leq M^{2} .
$$

This proves Proposition 4.5.

\section{$\S 5 . \quad$ Bootstrap Argument}

We have constructed a weak solution to the dispersive equation (2.3) satisfying the following extra conormal regularity:

$$
\left\|P^{k} u\right\|_{H^{s}} \leq C A_{1}^{k}(k-1) ! \quad k=0,1, \ldots,
$$

under the condition to the initial data $\phi$ :

$$
\left\|\left(x \partial_{x}\right)^{k} \phi\right\|_{H^{s}} \leq C A_{1}^{k} k ! \quad k=0,1, \ldots
$$

Introducing a smooth shut-off $a(x)=\langle x\rangle^{-2}$, where $\langle x\rangle=\left(1+|x|^{2}\right)^{-1}$, we firstly derive

$$
\left\|a^{l} \partial_{x}^{l} P^{k} u(t)\right\|_{H_{x}^{1}(\mathbb{R})} \leq C\left\langle t^{-1}\right\rangle^{l} A_{1}^{k+l}(k+l) ! \quad k, l=0,1,2, \ldots .
$$

To this end, we recall the following lemma originally due to Calderón [3] which plays a key role in the first step of the regularity bootstrap argument (see also [17] Lemma 2.1).

Lemma 5.1 ([3]). If $\left\|a^{l} \partial_{x}^{l} f\right\|_{2} \leq C A^{l} l !\|f\|_{2}$ for $0 \leq l \leq N-1$, then there exists a constant $A>0$ such that we have

$$
\left\|\left[\mathcal{H}_{x}, a^{N}\right] \partial_{x}^{N} f\right\|_{2} \leq C A^{N} N !\|f\|_{2}
$$

Proof of Lemma 5.1. Although the proof may be found in literature, we give a proof which is using the explicit expression of the commutator $\left[\mathcal{H}_{x}, a\right] .^{1}$ Let $f \in E_{x}$. Since

$$
\left\|\left[\mathcal{H}_{x}, a^{N}\right] \partial_{x}^{N} f\right\|_{2} \leq \sum_{j=0}^{N-1}\left\|a^{j}\left[\mathcal{H}_{x}, a\right] a^{N-1-j} \partial_{x}^{N} f\right\|_{2},
$$

\footnotetext{
${ }^{1}$ It is also possible to show the $N$ dependence of the operator norm of $\left[\mathcal{H}_{x}, a^{N}\right]$ directly by passing the Fourier transform.
} 
it suffices to show that

$$
\left\|a^{j}\left[\mathcal{H}_{x}, a\right] a^{N-1-j} \partial_{x}^{N} f\right\|_{2} \leq C A^{N}(N-1) !\|f\|_{2} .
$$

An elementary computation gives

$$
\left[\mathcal{H}_{x}, a\right] f=\text { p.v. } \int_{\mathbb{R}} \frac{a(y)-a(x)}{x-y} f(y) d y=\text { p.v. } \int_{\mathbb{R}} \frac{x+y}{\langle x\rangle^{2}\langle y\rangle^{2}} f(y) d y .
$$

By integration by parts, we have

$$
\begin{aligned}
\| a^{j} & {\left[\mathcal{H}_{x}, a\right] a^{N-1-j} \partial_{x}^{N} f \|_{2}^{2} } \\
= & \left.\int_{\mathbb{R}}\langle x\rangle^{-2 j} \int_{\mathbb{R}} \frac{x+y}{\langle x\rangle^{2}\langle y\rangle^{2}}\langle y\rangle^{-2(N-1-j)} \partial_{x}^{N} f(y) d y\right|^{2} d x \\
= & \iiint \frac{(x+y)(x+z)}{\langle x\rangle^{4(j+1)}}\langle y\rangle^{-2(N-j)}\langle z\rangle^{-2(N-j)} \partial_{y}^{N} f(y) \partial_{z}^{N} \bar{f}(z) d y d z d x \\
= & \iint \partial_{y}^{j+1} \partial_{z}^{j+1}\left\{\left(\int_{\mathbb{R}} \frac{(x+y)(x+z)}{\langle x\rangle^{4(j+1)}} d x\right)\langle y\rangle^{-2(N-j)}\langle z\rangle^{-2(N-j)}\right\} \\
& \quad \times \partial_{y}^{N-j-1} f(y) \partial_{z}^{N-j-1} \bar{f}(z) d y d z .
\end{aligned}
$$

If we set $\sigma(y, z)=\int_{\mathbb{R}}((x+y)(x+z)) /\left(\langle x\rangle^{4(j+1)}\right) d x=\sigma_{1}+\sigma_{0} y z$ and $\max \left(\sigma_{0}, \sigma_{1}\right)$ $=\tilde{\sigma}$, where $\sigma_{i}(i=0,1)$ are constants of order $j^{1 / 2}$, then

$$
\begin{aligned}
&\left|\partial_{y}^{j+1} \partial_{z}^{j+1}\left(\sigma(y, z)\langle y\rangle^{-2(N-j)}\langle z\rangle^{-2(N-j)}\right)\right| \\
& \leq \sigma_{0} j^{2} \partial_{y}^{j}\langle y\rangle^{-2(N-j)} \partial_{z}^{j}\langle z\rangle^{-2(N-j)}+\sigma_{0} j y \partial_{y}^{j+1}\langle y\rangle^{-2(N-j)} \partial_{z}^{j}\langle z\rangle^{-2(N-j)} \\
&+\sigma_{0} j z \partial_{y}^{j}\langle y\rangle^{-2(N-j)} \partial_{z}^{j+1}\langle z\rangle^{-2(N-j)} \\
&+\left(\sigma_{1}+\sigma_{0} y z\right) \partial_{y}^{j+1}\langle y\rangle^{-2(N-j)} \partial_{z}^{j+1}\langle z\rangle^{-2(N-j)} \\
& \leq C_{0} j^{2} A_{0}^{j+1}\left(\frac{2^{j} N !}{(N-j-1) !}\right)^{2}\langle y\rangle^{-2 N+j}\langle z\rangle^{-2 N+j} \\
&+\sigma_{0} j A_{0}^{j+1}\left(\frac{2^{j} N !}{(N-j-1) !}\right)\left(\frac{2^{j}(N+1) !}{(N-j-1) !}\right) \\
& \times\left\{\langle y\rangle\langle y\rangle^{-2 N+j-1}\langle z\rangle^{-2 N+j}+\langle z\rangle\langle y\rangle^{-2 N+j}\langle z\rangle^{-2 N+j-1}\right\} \\
&+\tilde{\sigma}\langle y\rangle\langle z\rangle A_{0}^{j+1}\left(\frac{2^{j}(N+1) !}{(N-j-1) !}\right)^{2}\langle y\rangle^{-2 N+j-1}\langle z\rangle^{-2 N+j-1} \\
& \leq C \tilde{\sigma}(j+1)^{2} A_{1}^{j+1}\left(\frac{N !}{(N-j-1) !}\right)^{2}\langle y\rangle^{-2 N+j}\langle z\rangle^{-2 N+j} .
\end{aligned}
$$


Hence it follows by the assumption that

$$
\begin{aligned}
& \left\|a^{j}\left[\mathcal{H}_{x}, a\right] a^{N-1-j} \partial_{x}^{N} f\right\|_{2}^{2} \\
& \quad \leq C \tilde{\sigma}(j+1)^{2} A_{1}^{j+1}\left(\frac{N !}{(N-j-1) !}\right)^{2}\left\|a^{N-j / 2} \partial_{x}^{N-j-1} f\right\|_{1}^{2} \\
& \quad \leq C \tilde{\sigma}(j+1)^{2} A_{1}^{j+1}\left(\frac{N !}{(N-j-1) !}\right)^{2}\left\|a^{j / 2+1}\right\|_{2}^{2}\left\|a^{N-j-1} \partial_{x}^{N-j-1} f\right\|_{2}^{2} \\
& \quad \leq C \tilde{\sigma}(j+1)^{2} A_{1}^{j+1} A_{1}^{2(N-j-1)}(N !)^{2}\|f\|_{2}^{2} \\
& \quad \leq 4^{-(j+1)} C^{2} A^{2 N}(N !)^{2}\|f\|_{2}^{2}
\end{aligned}
$$

and we conclude

$$
\left\|\left[\mathcal{H}_{x}, a^{N}\right] f\right\|_{2} \leq C A^{N} N ! \sum_{j=1}^{N-1} 2^{-(j+1)}\|f\|_{2} \leq C A^{N} N !\|f\|_{2} .
$$

Based upon the above Lemma 5.1, we proceed to show the regularity. The first step is the following proposition.

Proposition 5.2. $\quad$ Let $u$ be a solution constructed in Proposition 4.1. That is for $k=0,1,2, \ldots,\left\|P^{k} u(t)\right\|_{H^{s}} \leq C A^{k} k$ !. Then we have

$$
\left\|\langle x\rangle^{-2 l} \partial_{x}^{l} P^{k} u(t)\right\|_{H^{1}} \leq C_{3}\left\langle t^{-1}\right\rangle^{l} A_{1}^{k+l}(k+l) !
$$

for all $k, l=0,1,2, \ldots$.

Proof of Proposition 5.2. As in the former context, we set the weight function $\langle x\rangle^{-2}$ as $a(x)$. We prove the Proposition by an induction with respect to $l$. For $l=0$, the equation (5.3) is nothing but the assumption. Let we assume that for $0 \leq m \leq l$

$$
\left\|a^{m} \partial_{x}^{m} P^{k} u\right\|_{H^{1}} \leq C\left\langle t^{-1}\right\rangle^{m} A_{0}^{k+m}(k+m) ! \quad \text { for all } k=0,1,2, \ldots
$$

We claim for some $A_{1}>0$ which is determined independently on $k$ and $l$ such that

$$
\left\|a^{l+1} \partial_{x}^{l+1} P^{k} u\right\|_{H^{1}} \leq C\left\langle t^{-1}\right\rangle^{l+1} A_{1}^{k+l+1}(k+l+1) ! \quad \text { for all } k=0,1,2, \ldots .
$$

The $H^{1}$ norm can be divided into two parts:

$$
\begin{aligned}
\left\|a^{l+1} \partial_{x}^{l+1} P^{k} u\right\|_{H^{1}} & \leq\left\|a^{l+1} \partial_{x}^{l+1} P^{k} u\right\|_{2}+\left\|\partial_{x} a^{l+1} \partial_{x}^{l+1} P^{k} u\right\|_{2} \\
& \equiv I_{1}+I_{2} .
\end{aligned}
$$


Since

$$
\left|\partial_{x} a^{l}\right|=\left|\partial_{x}\langle x\rangle^{-2 l}\right| \leq \frac{2 l|x|}{\langle x\rangle^{2(l+1)}} \leq 2 l a^{l+1 / 2}(x),
$$

it follows from the assumption that

$$
\begin{aligned}
I_{1} & \leq\left\|a^{l} \partial_{x}^{l+1} P^{k} u\right\|_{2} \leq\left\|\partial_{x} a^{l} \partial_{x}^{l} P^{k} u\right\|_{2}+\left\|\left(\partial_{x} a^{l}\right) \partial_{x}^{l} P^{k} u\right\|_{2} \\
& \leq\left\|a^{l} \partial_{x}^{l} P^{k} u\right\|_{H^{1}}+2 l\left\|a^{l+1 / 2} \partial_{x}^{l} P^{k} u\right\|_{2} \\
& \leq\left\|a^{l} \partial_{x}^{l} P^{k} u\right\|_{H^{1}}+2 l\left\|a^{l} \partial_{x}^{l} P^{k} u\right\|_{2} \\
& \leq(1+2 l)\left\|a^{l} \partial_{x}^{l} P^{k} u\right\|_{H^{1}} \leq C(1+2 l)\left\langle t^{-1}\right\rangle^{l} A_{0}^{k+l}(k+l) ! \\
& \leq 2 C\left\langle t^{-1}\right\rangle^{l} A_{0}^{k+l}(k+l+1) !
\end{aligned}
$$

for $k=0,1,2, \ldots$.

To the second term in (5.5), we use (5.6) with $l+1$ instead of $l$ and obtain

$$
\begin{aligned}
I_{2} \leq & \left\|\partial_{x}\left(a^{l+1}\right) \partial_{x}^{l+1} P^{k} u\right\|_{2}+\left\|a^{l+1} \partial_{x}^{l+2} P^{k} u\right\|_{2} \\
\leq & 2(l+1)\left\|a^{l+3 / 2} \partial_{x}^{l+1} P^{k} u\right\|_{2}+\left\|H_{x} a^{l+1} \partial_{x}^{l+2} P^{k} u\right\|_{2} \\
\leq & 2(l+1)\left\{\left\|\partial_{x} a^{l} \partial_{x}^{l} P^{k} u\right\|_{2}+\left\|\left(\partial_{x} a^{l}\right) \partial_{x}^{l} P^{k} u\right\|_{2}\right\} \\
& +\left\|\left[H_{x}, a^{l+1}\right] \partial_{x}^{l+2} P^{k} u\right\|_{2}+\left\|a^{l+1} \partial_{x}^{l} H_{x} \partial_{x}^{2} P^{k} u\right\|_{2} \\
\equiv & J_{1}+J_{2}+J_{3} .
\end{aligned}
$$

Now the first term $J_{1}$ of the right hand side in (5.8) is bounded via (5.6) by

$$
\begin{aligned}
& 2(l+1)\left\{\left\|\partial_{x} a^{l} \partial_{x}^{l} P^{k} u\right\|_{2}+2 l\left\|a^{l+1 / 2} \partial_{x}^{l} P^{k} u\right\|_{2}\right\} \\
& \quad \leq 2(l+1)\left\{\left\|a^{l} \partial_{x}^{l} P^{k} u\right\|_{H^{1}}+2 l\left\|a^{l+1 / 2} \partial_{x}^{l} P^{k} u\right\|_{2}\right\} \\
& \quad \leq C(l+1)^{2}\left\langle t^{-1}\right\rangle^{l} A_{0}^{k+l}(k+l) ! \\
& \quad \leq C\left\langle t^{-1}\right\rangle^{l} A_{0}^{k+l}(k+l+2) ! \leq C\left\langle t^{-1}\right\rangle^{l} A_{1}^{k+l}(k+l+1) ! .
\end{aligned}
$$

The commutator term $J_{2}$ in (5.8) is dealt with Lemma 5.1 that

$$
\begin{aligned}
J_{2} & \leq C A_{1}^{l+1}(l+1) !\left\|\partial_{x} P^{k} u\right\|_{2} \leq C A_{1}^{l+1}(l+1) !\left\|P^{k} u\right\|_{H^{1}} \\
& \leq C A_{1}^{l+1} A_{0}^{k}(l+1) ! k ! \leq C A_{1}^{k+l+1}(k+l+1) ! .
\end{aligned}
$$

For the last term $J_{3}$ we invoke the equation that $P^{k} u$ satisfies.

$$
H_{x} \partial_{x}^{2} u_{k}=-\frac{1}{2 t} P u_{k}+\frac{1}{2 t} x \partial_{x} u_{k}+B_{k}(u, u),
$$


where $u_{k}=P^{k} u$. Substituting the above equation, we divide $J_{3}$ as the following:

$$
\text { (5.11) } \begin{aligned}
J_{3} & \leq \frac{1}{2|t|}\left\|a^{l+1} \partial_{x}^{l} P^{k+1} u\right\|_{2}+\frac{1}{2|t|}\left\|a^{l+1} \partial_{x}^{l} x \partial_{x} P^{k} u\right\|_{2}+\left\|a^{l+1} \partial_{x}^{l} B_{k}(u, u)\right\|_{2} \\
& \leq J_{3}^{1}+J_{3}^{2}+J_{3}^{3} .
\end{aligned}
$$

The first and second terms in the right hand side in (5.11) are estimated easily by the assumption (5.4).

$$
J_{3}^{1} \leq \frac{1}{2|t|}\left\|a^{l} \partial_{x}^{l} P^{k+1} u\right\|_{2} \leq C|t|^{-1}\left\langle t^{-1}\right\rangle^{l} A_{0}^{k+l+1}(k+l+1) ! .
$$

From (5.6),

$$
\begin{aligned}
J_{3}^{2} & \leq(2|t|)^{-1}\left\{\left\|a^{l+1} x \partial_{x}^{l+1} P^{k} u\right\|_{2}+l\left\|a^{l+1} \partial_{x}^{l} P^{k} u\right\|_{2}\right\} \\
& \leq(2|t|)^{-1}\left\{\left\|a^{l} \partial_{x}^{l+1} P^{k} u\right\|_{2}+l\left\|a^{l} \partial_{x}^{l} P^{k} u\right\|_{2}\right\} \\
& \leq(2|t|)^{-1}\left\{\left\|\partial_{x} a^{l} \partial_{x}^{l} P^{k} u\right\|_{2}+\left\|\left(\partial_{x} a^{l}\right) \partial_{x}^{l} P^{k} u\right\|_{2}+l\left\|a^{l} \partial_{x}^{l} P^{k} u\right\|_{2}\right\} \\
& \leq(2|t|)^{-1}\left\|a^{l} \partial_{x}^{l} P^{k} u\right\|_{H^{1}}+|t|^{-1}\left\|a^{l+1 / 2} \partial_{x}^{l} P^{k} u\right\|_{2} \\
& \leq C(l+1)|t|^{-1}\left\|a^{l} \partial_{x}^{l} P^{k} u\right\|_{H^{1}} \leq C(l+1)|t|^{-1}\left\langle t^{-1}\right\rangle^{l} A_{0}^{k+l}(k+l) ! \\
& \leq C|t|^{-1}\left\langle t^{-1}\right\rangle^{l} A_{0}^{k+l}(k+l+1) !
\end{aligned}
$$

For the third term involving the nonlinear interaction, we recall its structure (2.1) and proceed the estimate as follows.

$$
\begin{aligned}
J_{3}^{3} & \leq\left\|a^{l} \partial_{x}^{l} B_{k}(u, u)\right\|_{2} \\
& \leq \sum_{k=k_{0}+k_{1}+k_{2}} \frac{k !}{k_{0} ! k_{1} ! k_{2} !}\left\|a^{l} \partial_{x}^{l+1}\left(u_{k_{1}} u_{k_{2}}\right)\right\|_{2} \\
& \leq \sum_{k=k_{0}+k_{1}+k_{2}} \frac{k !}{k_{0} ! k_{1} ! k_{2} !} \sum_{m=0}^{l} \frac{l !}{m !(l-m) !}\left\|a^{l} \partial_{x}\left(\partial_{x}^{m} u_{k_{1}} \partial_{x}^{l-m} u_{k_{2}}\right)\right\|_{2} \\
& \leq \sum_{k=k_{0}+k_{1}+k_{2}} \frac{k !}{k_{0} ! k_{1} ! k_{2} !} \sum_{m=0}^{l} \frac{l !}{m !(l-m) !}\left\|a^{m} \partial_{x}^{m} u_{k_{1}}\right\|_{H^{1}}\left\|a^{l-m} \partial_{x}^{l-m} u_{k_{2}}\right\|_{H^{1}} .
\end{aligned}
$$

Here we employed the Hölder inequality and a similar commuting argument in (5.13). By the assumption, it follows from (5.14) and by changing $k_{0}=k-k^{\prime}$, 
$k_{1}=k^{\prime}-k^{\prime \prime}, k_{2}=k^{\prime \prime}$ that

$$
\begin{aligned}
J_{3}^{3} \leq & C \sum_{k=k_{0}+k_{1}+k_{2}} \frac{k !}{k_{0} ! k_{1} ! k_{2} !} \\
& \times \sum_{m=0}^{l} \frac{l !}{m !(l-m) !}\left(m+k_{1}\right) !\left(l-m+k_{2}\right) !\left\langle t^{-1}\right\rangle^{l} A_{0}^{l+k-k_{0}} \\
= & C \sum_{k^{\prime}=0}^{k} \sum_{k^{\prime \prime}=0}^{k^{\prime}} \frac{k !}{\left(k-k^{\prime}\right) !\left(k^{\prime}-k^{\prime \prime}\right) ! k^{\prime \prime} !} \\
& \times \sum_{m=0}^{l} \frac{l !}{m !(l-m) !}\left(m+k^{\prime}-k^{\prime \prime}\right) !\left(l-m+k^{\prime \prime}\right) !\left\langle t^{-1}\right\rangle^{l} A_{0}^{l+k^{\prime}} \\
\leq & C \sum_{k^{\prime}=0}^{k} \sum_{k^{\prime \prime}=0}^{k^{\prime}} \frac{k !}{\left(k-k^{\prime}\right) !\left(k^{\prime}-k^{\prime \prime}\right) ! k^{\prime \prime} !}\left(\sum_{m=0}^{l} \frac{l !}{m !(l-m) !}\right)\left(l+k^{\prime}\right) !\left\langle t^{-1}\right\rangle^{l} A_{0}^{l+k^{\prime}} \\
\leq & C \sum_{k^{\prime}=0}^{k} \frac{k !}{\left(k-k^{\prime}\right) ! k^{\prime} !}\left(\sum_{k^{\prime \prime}=0}^{k^{\prime}} \frac{k^{\prime} !}{\left(k^{\prime}-k^{\prime \prime}\right) ! k^{\prime \prime !}}\right) 2^{l}\left(l+k^{\prime}\right) !\left\langle t^{-1}\right\rangle^{l} A_{0}^{l+k^{\prime}} \\
= & C \sum_{k^{\prime}=0}^{k} \frac{k !}{\left(k-k^{\prime}\right) ! k^{\prime} !}\left(2 A_{0}\right)^{l+k^{\prime}}\left\langle t^{-1}\right\rangle^{l}(l+k) ! \\
= & C\left\langle t^{-1}\right\rangle^{l}\left(2 A_{0}+1\right)^{k+l}(l+k) ! \leq C\left\langle t^{-1}\right\rangle^{l} A_{1}^{k+l}(k+l) !
\end{aligned}
$$

Hence by gathering the estimates (5.11)-(5.13) and (5.15) and setting the constant $A_{1}$ larger, we have

$$
J_{3} \leq J_{3}^{1}+J_{3}^{2}+J_{3}^{3} \leq C\left(1+|t|^{-1}\right)\left\langle t^{-1}\right\rangle^{l} A_{1}^{k+l+1}(k+l+1) !
$$

By changing the constants $C$ and $A_{1}$, if necessary, we together estimates (5.5), (5.7), (5.8)-(5.10) and (5.16) to conclude that

$$
\left\|a^{l+1} \partial_{x}^{l+1} P^{k} u\right\|_{H^{1}} \leq C\left\langle t^{-1}\right\rangle^{l+1} A_{1}^{k+l+1}(k+l+1) ! \quad k, l=0,1,2, \ldots
$$

Rest of the proof goes a similar way as in [25]. By the Sobolev embedding theorem, we have from Proposition 5.2 that for any $x_{0} \in \mathbb{R}$ and some $\delta>0$

$$
\left\|\partial_{x}^{l} P^{k} u(t)\right\|_{L^{\infty}\left(I_{x_{0}}\right)} \leq C\left\langle t^{-1}\right\rangle^{l}\left\langle x_{0}\right\rangle^{2 l} A_{1}^{k+l}(k+l) ! \quad k, l=0,1,2, \ldots,
$$


where $I_{x_{0}}=\left(x_{0}-\delta, x_{0}+\delta\right)$. From this point wise estimate, we forward the second step and the operator $P$ can be translated into the time derivative via $t \partial_{t}=(1 / 2)\left(P-x \partial_{x}\right)$.

$$
\sup _{t}\left\|a\left(t \partial_{t}\right)^{l_{1}} \partial_{x}^{l_{2}} u\right\|_{H_{x}^{1}(\mathbb{R})} \leq C A_{2}^{l_{1}+l_{2}}\left(l_{1}+l_{2}\right) ! \quad l_{1}, l_{2}=0,1,2, \ldots
$$

This gives the regularity for the solution.

Proposition 5.3. For $\delta>0$, we denote $I_{x_{0}}=\left(x_{0}-\delta, x_{0}+\delta\right)$. Suppose that there exist positive constants $C$ and $A_{4}$ such that

$$
\begin{aligned}
\sup _{t \in\left[t_{0}-\delta, t_{0}+\delta\right]}\left\|\partial_{x}^{l} P^{k} u(t)\right\|_{L^{\infty}\left(I_{x_{0}}\right)} & \\
& \leq C_{0}\left\langle t_{0}^{-1}\right\rangle^{l}\left\langle x_{0}\right\rangle^{2 l} A_{1}^{k+l}(k+l) ! \quad k, l=0,1,2, \ldots .
\end{aligned}
$$

Then we have

$$
\begin{aligned}
\sup _{t \in\left[t_{0}-\delta, t_{0}+\delta\right]}\left\|\partial_{t}^{j} \partial_{x}^{l} u(t)\right\|_{L^{\infty}\left(I_{x_{0}}\right)} & \\
& \leq C\left\langle t_{0}^{-1}\right\rangle^{j+l}\left\langle x_{0}\right\rangle^{2 l+3 j} A_{2}^{j+l}(j+l) ! \quad j, l=0,1,2, \ldots,
\end{aligned}
$$

where the constants $C$ and $A_{2}$ only depend on $C_{0}, A_{1}, \delta$ and $I_{x_{0}}$.

Proof of Proposition 5.3. In the following proof, we fix the point $x_{0}$ and the interval $I_{x_{0}}$. First we claim that for some positive constants $C, A_{2}$ and $B_{1}$

$$
\left\|\left(x \partial_{x}\right)^{m} \partial_{x}^{l} P^{k} u(t)\right\|_{L^{\infty}\left(I_{x_{0}}\right)} \leq C\left\langle t^{-1}\right\rangle^{l}\left\langle x_{0}\right\rangle^{2 l+3 m} A_{2}^{k+m+l} B_{1}^{m}(k+m+l) !
$$

for $k, l, m=0,1,2, \ldots$ For a while every constant depending on $\left\langle t^{-1}\right\rangle$ is simply denoted as $C$.

We again use an induction argument: Suppose that (5.17) is valid up to $m$.

$$
\begin{aligned}
& \left\|\left(x \partial_{x}\right)^{m+1} \partial_{x}^{l} P^{k} u\right\|_{L^{\infty}\left(I_{x_{0}}\right)} \\
& \quad=\left\|x \partial_{x}\left(x \partial_{x}\right)^{m} \partial_{x}^{l} P^{k} u\right\|_{L^{\infty}\left(I_{x_{0}}\right)} \\
& \quad \leq C\left\langle x_{0}\right\rangle\left\|\left(x \partial_{x}+1\right)^{m} \partial_{x}^{l+1} P^{k} u\right\|_{L^{\infty}\left(I_{x_{0}}\right)} \\
& \quad \leq C\left\langle x_{0}\right\rangle \sum_{m_{1}=1}^{m} \frac{m !}{m_{1} !\left(m-m_{1}\right) !}\left\|\left(x \partial_{x}\right)^{m_{1}} \partial_{x}^{l+1} P^{k} u\right\|_{L^{\infty}\left(I_{x_{0}}\right)} \\
& \quad \leq C\left\langle x_{0}\right\rangle \sum_{m_{1}=1}^{m} \frac{m !}{m_{1} !\left(m-m_{1}\right) !}
\end{aligned}
$$




$$
\begin{aligned}
& \times\left\langle x_{0}\right\rangle^{2(l+1)+3 m_{1}} A_{2}^{k+m_{1}+l+1} B_{1}^{m_{1}}\left(k+m_{1}+l+1\right) ! \\
\leq & C\left\langle x_{0}\right\rangle^{2(l+1)+3 m+1} A_{2}^{k+m+l+1} B_{1}^{m}(k+m+l+1) ! \\
& \times \sum_{m_{1}=1}^{m} \frac{\left\langle x_{0}\right\rangle^{-3\left(m-m_{1}\right)}\left(A_{2} B_{1}\right)^{-\left(m-m_{1}\right)}}{\left(m-m_{1}\right) !} \frac{m !}{m_{1} !} \frac{\left(k+m_{1}+l+1\right) !}{(k+m+l+1) !} \\
\leq & C e^{-A_{2} B_{1}\left\langle x_{0}\right\rangle^{3}}\left\langle x_{0}\right\rangle^{2 l+3(m+1)} A_{2}^{k+m+l+1} B_{1}^{m}(k+m+l+1) !,
\end{aligned}
$$

where we take $B_{2}$ so large that $B_{2} \geq \max \left(e^{-A_{2} B_{1}\left\langle x_{0}\right\rangle^{3}}, 1\right)$. Note that this choice of $B_{2}$ is independent of $x_{0}$.

The second claim is that for some positive constants $C=C\left(\left\langle t^{-1}\right\rangle\right), A_{3}$ such that

$$
\left\|\left(t \partial_{t}\right)^{m} \partial_{x}^{l} u(t)\right\|_{L^{\infty}\left(I_{x_{0}}\right)} \leq C\left\langle x_{0}\right\rangle^{2 l+3 m} A_{3}^{l+m}(l+m) ! \quad(l, m=0,1,2, \ldots) .
$$

Since $t \partial_{t}=(1 / 2)\left(P-x \partial_{x}\right)$, we have

$$
\begin{aligned}
& \left\|\left(t \partial_{t}\right)^{m} \partial_{x}^{l} u\right\|_{L^{\infty}\left(I_{x_{0}}\right)} \\
& \quad \leq 2^{-m} \sum_{m=m_{1}+m_{2}} \frac{m !}{m_{1} ! m_{2} !}\left\|\left(x \partial_{x}\right)^{m_{1}} P^{m_{2}} \partial_{x}^{l} u\right\|_{L^{\infty}\left(I_{x_{0}}\right)} \\
& \quad=2^{-m} \sum_{m=m_{1}+m_{2}} \frac{m !}{m_{1} ! m_{2} !}\left\|\left(x \partial_{x}\right)^{m_{1}} \partial_{x}^{l}(P-l)^{m_{2}} u\right\|_{L^{\infty}\left(I_{x_{0}}\right)}
\end{aligned}
$$

(by replacing $m_{2}$ into $m_{2}+m_{3}$ ),

$$
\leq 2^{-m} \sum_{m=m_{1}+m_{2}+m_{3}} \frac{m !}{m_{1} ! m_{2} ! m_{3} !} l^{m_{3}}\left\|\left(x \partial_{x}\right)^{m_{1}} \partial_{x}^{l} P^{m_{2}} u\right\|_{L^{\infty}\left(I_{x_{0}}\right)} .
$$

By the induction assumption, we have with $B_{2}=A_{2} B_{1}(\geq 1)$ that

$$
\begin{aligned}
& \left\|\left(t \partial_{t}\right)^{m} \partial_{x}^{l} u\right\|_{L^{\infty}\left(I_{x_{0}}\right)} \\
& \leq 2^{-m} \sum_{m=m_{1}+m_{2}+m_{3}} \frac{m !}{m_{1} ! m_{2} ! m_{3} !} l^{m_{3}} C\left\langle x_{0}\right\rangle^{2 l+3 m_{1}} B_{2}^{m_{1}+m_{2}+l}\left(m_{1}+m_{2}+l\right) ! \\
& \leq 2^{-m} C\left\langle x_{0}\right\rangle^{2 l} B_{2}^{m+l}(m+l) ! \\
& \quad \times \sum_{m=m_{1}+m_{2}+m_{3}} \frac{m !}{m_{1} ! m_{2} ! m_{3} !}\left\langle x_{0}\right\rangle^{3 m_{1}} B_{2}^{-m_{3}} l^{m_{3}} \frac{\left(m_{1}+m_{2}+l\right) !}{(m+l) !} .
\end{aligned}
$$

Observing that

$$
l^{m_{3}} \frac{\left(m_{1}+m_{2}+l\right) !}{(m+l) !} \leq 1
$$

we see from (5.18) that

$$
\left\|\left(t \partial_{t}\right)^{m} \partial_{x}^{l} u\right\|_{L^{\infty}\left(I_{x_{0}}\right)} \leq 2^{-m} C\left\langle x_{0}\right\rangle^{2 l}\left(1+\left\langle x_{0}\right\rangle^{3}+B_{2}^{-1}\right)^{m} B_{2}^{l+m}(l+m) !
$$




$$
\leq C\left\langle x_{0}\right\rangle^{2 l+3 m} A_{3}^{l+m}(l+m) !
$$

Finally, we give a process to remove $t$ in the time derivative in (5.19). To this end, we show that for some positive constants $C, A_{4}$ and $B_{3}$ we have

$$
\left\|\left(t \partial_{t}\right)^{m} \partial_{t}^{j} \partial_{x}^{l} u\right\|_{L^{\infty}\left(I_{x_{0}}\right)} \leq C\left\langle t^{-1}\right\rangle^{l+j}\left\langle x_{0}\right\rangle^{2 l+3 m+3 j} A_{4}^{j+m+l} B_{3}^{j}(j+m+l) !,
$$

for $j, l, m=0,1,2, \ldots$. We show this by induction with respect to $m$. Obviously (5.20) is valid for $m=0$. We assume that (5.20) holds for $m$. Then it follows

$$
\begin{aligned}
& \left\|\left(t \partial_{t}\right)^{m} \partial_{t}^{j+1} \partial_{x}^{l} u(t)\right\|_{L^{\infty}\left(I_{x_{0}}\right)} \\
& \quad \leq\left\|\partial_{t}\left(t \partial_{t}-1\right)^{m} \partial_{t}^{j} \partial_{x}^{l} u\right\|_{L^{\infty}\left(I_{x_{0}}\right)} \\
& \quad=|t|^{-1}\left\|t \partial_{t}\left(t \partial_{t}-1\right)^{m} \partial_{t}^{j} \partial_{x}^{l} u\right\|_{L^{\infty}\left(I_{x_{0}}\right)} \\
& \quad \leq|t|^{-1} \sum_{m_{1}=0}^{j} \frac{m !}{m_{1} !\left(m-m_{1}\right) !}\left\|\left(t \partial_{t}\right)^{m_{1}+1} \partial_{t}^{j} \partial_{x}^{l} u\right\|_{L^{\infty}\left(I_{x_{0}}\right)}
\end{aligned}
$$

Again by the induction assumption, we have

$$
\begin{aligned}
\|( & \left.t \partial_{t}\right)^{m} \partial_{t}^{j+1} \partial_{x}^{l} u(t) \|_{L^{\infty}\left(I_{x_{0}}\right)} \\
\leq & C|t|^{-1} \sum_{m_{1}=0}^{j} \frac{m !}{m_{1} !\left(m-m_{1}\right) !} \\
& \times\left\langle t^{-1}\right\rangle^{l+j}\left\langle x_{0}\right\rangle^{2 l+3 j+3\left(m_{1}+1\right)} A_{4}^{j+m_{1}+l+1} B_{3}^{j}\left(j+m_{1}+l+1\right) ! \\
= & C|t|^{-1}\left\langle t^{-1}\right\rangle^{l+j}\left\langle x_{0}\right\rangle^{2 l+3 j+3(m+1)} A_{4}^{j+m+l+1} B_{3}^{j}(j+m+l+1) ! \\
& \times \sum_{m_{1}=0}^{m} \frac{A_{4}^{-\left(m-m_{1}\right)}\left\langle x_{0}\right\rangle^{-3\left(m-m_{1}\right)}}{\left(m-m_{1}\right) !} \frac{\left.m+m_{1}+l+1\right) !}{m_{1} !(j+m+l+1) !} \\
= & C|t|^{-1}\left\langle t^{-1}\right\rangle^{l+j} e^{-A_{4}\left\langle x_{0}\right\rangle^{3}}\left\langle x_{0}\right\rangle^{2 l+3 m+3(j+1)} A_{4}^{j+m+l+1} B_{3}^{j}(j+m+l+1) ! \\
\leq & C\left\langle t^{-1}\right\rangle^{l+j+1}\left\langle x_{0}\right\rangle^{2 l+3 m+3(j+1)} A_{4}^{j+m+l+1} B_{3}^{j+1}(j+m+l+1) !,
\end{aligned}
$$

where we take $B_{3}$ larger than $e^{-A_{4}}$. Finally we choose $m=0$ in (5.20) and take $A_{5}=A_{4} B_{3}$ for obtaining the conclusion of proposition. This completes the proof of analyticity.

Remark 5. As is seen in the above proof, one could directly apply our argument for a solution in a class of $C\left(\mathbb{R} ; H^{1}(\mathbb{R})\right)$ once the well-posedness in that class is established. It is known that there is a weak global solution in the class $H^{1}$. However it is not clear if the well-posedness, especially the uniqueness of a weak solution holds. See for example [35]. 
The proof of Theorem 1.2 can be shown by very much similar way and we left it to reader.

\section{§6. Appendix}

Here we give the explicit proof of $\left\|\mathcal{F}\left[\partial_{x}\langle x\rangle^{\delta}\right]\right\|_{1}<\infty$ which is required in the proof of Lemma 4.3. From the definition of the $\Gamma$-function, we have

$$
\langle x\rangle^{\delta-2}=\frac{1}{\Gamma(a)} \int_{0}^{\infty} e^{-\lambda\left(1+x^{2}\right)} \lambda^{-\delta / 2} d \lambda,
$$

where $\delta=1 / 2+\sigma$ and $a=(2-\delta) / 2>0$.

Since

$$
\mathcal{F}\left[\langle x\rangle^{\delta-2}\right]=\frac{1}{\sqrt{4 \pi} \Gamma(a)} \int_{0}^{\infty} e^{-\lambda} e^{-\frac{\xi^{2}}{4 \lambda}} \lambda^{-\delta / 2-1 / 2} d \lambda,
$$

it follows by the explicit computation that

$$
\begin{aligned}
\left\|\widehat{\partial_{x}\langle x\rangle^{\delta}}\right\|_{1} & =\left\|\widehat{\delta x\langle x\rangle^{\delta}-2}\right\|_{1}=\delta\left\|i \partial_{\xi} \mathcal{F}\left[\langle x\rangle^{\delta-2}\right]\right\|_{1} \\
& =\frac{\delta}{\sqrt{4 \pi} \Gamma(a)} \int_{\mathbb{R}}\left|\frac{1}{2} \int_{0}^{\infty} e^{-\lambda} \xi e^{-\frac{\xi^{2}}{4 \lambda}} \lambda^{-\delta / 2-3 / 2} d \lambda\right| d \xi \\
& =\frac{\delta}{\sqrt{4 \pi} \Gamma(a)} \int_{0}^{\infty} e^{-\lambda} \lambda^{-\delta / 2-3 / 2}\left(\int_{0}^{\infty} r e^{-\frac{r^{2}}{4 \lambda}} d r\right) d \lambda \\
& =\frac{4 \delta}{\sqrt{4 \pi} \Gamma(a)}\left(\int_{0}^{\infty} s e^{-s^{2}} d s\right) \int_{0}^{\infty} e^{-\lambda} \lambda^{-\delta / 2-1 / 2} d \lambda \\
& =\frac{\delta \Gamma(a+1)}{\sqrt{\pi} \Gamma(a)}=\frac{\delta}{\sqrt{\pi}}\left(1-\frac{\delta}{2}\right)
\end{aligned}
$$

provided $\delta<1$.

\section{Acknowledgement}

This work was originated when the second and forth authors attending the Short Term Collaboration Research project of RIMS, Kyoto University, during 1998 and 1999. K. Kato and T. Ogawa are grateful of the hospitality of RIMS, Kyoto University. Part of this work was done while the second author visited Departmento de Ciencias Básicas, Instituto Tecnológico de Morelia. He would like to thank for their kind hospitality and financial support. The work of E. Kaikina is supported by Scientific Research Project 28471-E by CONACYT-Mexico. E. I. Kaikina and P. I. Naumkin are partially supported by CONACYT-Mexico for the financial support. K. Kato is partially supported 
by JSPS grant-in-aid for scientific research, \#12740114. K. Kato and T. Ogawa are partially supported by JSPS grant-in-aid for scientific research, \#11440057.

\section{References}

[1] Bekiranov, D., Ogawa, T. and Ponce, G., Interaction Equations for Short and Long Dispersive Waves, J. Funct. Anal., 158 (2) (1998), 357-388.

[2] Benjamin, T. B., Internal waves of permanent form in fluids of great depth, J. Fluid. Mech., 29 (2) (1967), 559-592.

[3] Calderón, A. P., Commutators of singular integral operators, Proc. Nat. Acad. Sci. USA, 53 (1965), 1092-1099.

[4] Chihara, H., The initial value problem for cubic semilinear Schrödinger equations, Publ. RIMS, Kyoto Univ., 32 (1996), 445-471.

[5] — Gain of regularity for semilinear Schrödinger equations, Math. Ann., 315 (1999), 529-567.

[6] Constantin, P. and Saut, J. C., Local smoothing properties of dispersive equations, $J$. Amer. Math. Soc., 1 (1988), 413-446.

[7] de Bouard, A., Analytic solutions to non-elliptic nonlinear Schrödinger equations, $J$. Differential. Equations, 104 (1993), 196-213.

[8] de Bouard, A., Hayashi, N. and Kato, K., Regularizing effect for the (generalized) Korteweg-de Vries equation and nonlinear Schrödinger equations, Ann. Inst. H. Poincaré, Anal. Non Linéaire, 9 (1995), 673-725.

[9] Doi, S., On the Cauchy problem for Schrödinger type equations and the regularity of solutions, J. Math. Kyoto Univ., 34 (1994), 319-328.

[10] Ginibre, J. and Tsutsumi, Y., Uniqueness of solutions for the generalized Korteweg-de Vries equation, SIAM, J. Math. Anal., 20 (1989), 1388-1425.

[11] Ginibre, J. and Velo, G., Smoothing properties and existence of solutions for the generalized Benjamin-Ono equation, J. Differential Equations, 93 (1991), 150-212.

[12] Hayashi, N., Global existence of small analytic solutions to nonlinear Schrödinger equations, Duke Math. J., 60 (1990), 717-727.

[13] — The initial value problem for the derivative nonlinear Schrödinger equation in the energy space, Nonlinear Anal., 20 (1993), 823-833.

[14] - Local existence in time of solutions to the elliptic-hyperbolic Davey-Stewartson system without smallness condition on the data, J. Anal. Math., 73 (1997), 133-164.

[15] Hayashi, N. and Kato, K., Regularity in time of solutions to nonlinear Schrödinger equations, J. Funct. Anal., 128 (1995), 253-277.

[16] — Analyticity in time and smoothing effect of solutions to nonlinear Schrödinger equations, Comm. Math. Phys., 184 (1997), 273-300.

[17] Hayashi, N., Kato, K. and Ozawa, T., Dilation method and smoothing effects of solution to the Benjamin-Ono equation, Proc. Royal Soc. Edinburgh, 126A (1996), 273-285.

[18] Hayashi, N., Nakamitsu, K. and Tsutsumi, M., On solutions of the initial value problem for the nonlinear Schrödinger equations, J. Funct. Anal., 71 (1987), 218-245.

[19] Hayashi, N. and Naumkin, P., On the Davey-Stewartson and Ishimori systems, Math. Phys. Anal. Geom., 2 (1999), 53-81.

[20] Hayashi, N., Naumkin, P. and Pipolo, P. O., Analytic smoothing effect for some derivative nonlinear Schrödinger equations, Tsukuba J. Math., to appear.

[21] Hayashi, N. and Ozawa, T., Remarks on nonlinear Schrödinger equations in one space dimension, Differential Integral Equations, 7 (2) (1994), 453-461.

[22] Iorio Jr., R. J., On the Cauchy problem for the Benjamin-Ono equation, Comm. Partial Differential Equations, 11 (10) (1986), 1031-1081.

[23] Kappeler, T., Solutions to the Kortewe-de Vries equation with irregular initial profile, Comm. P.D.E., 11 (1986), 927-945. 
[24] Kajitani, K. and Wakabayashi, S., Analytically smoothing effect for Schrödinger type equations with variable coefficients, Preprint, Tsukuba University.

[25] Kato, K. and Ogawa, T., Analyticity and Smoothing Effect for the Korteweg-de Vries Equation with a single point singularity, Math. Ann., 316 (2000), 577-608.

[26] — Analytic smoothing effect and single point singularity for the nonlinear Schrödinger Equations, J. Korean Math. Soc., 37 (2000), 1071-1084.

[27] — Analytic smoothing effect and single point conormal regularity for the semilinear dispersive type equations, Joint Project report in RIMS Kyoto University, RIMS, Kôkyûroku, 1123 (2000), 112-123.

[28] Kato, K. and Taniguchi, K., Gevrey regularizing effect for nonlinear Schrödinger equations, Osaka J. Math., 33 (1996), 863-880.

[29] Kato, T., Quasilinear Equations of Evolutions, with applications to partial differential equations, Lecture Notes in Math., 448 (1975), 27-50.

[30] — - On the Cauchy problem for the (generalized) Korteweg-de Vries equation, in: "Studies in Applied Mathematics", edited by V. Guillemin, (ed.), Adv. Math. Supplementary Studies, 18 Academic Press 1983, 93-128.

[31] Kato, T. and Masuda, K., Nonlinear evolution equations and analyticity I, Ann. Inst. H. Poincaré Anal. Non Linéaire, 3 (1986), 455-467.

[32] Kato, T. and Ponce, G., Commutator estimate for Navier-Stokes equations, Comm. P.A.M., 3 (1986), 455-467.

[33] Kenig, C. E., Ponce, G. and Vega, L., Well-posedness and scattering results for the generalized Korteweg-de Vries equation via the contraction mapping principle, Comm. Pure Appl. Math., 46 (1993), 527-620.

[34] - The Cauchy problem for the Korteweg-de Vries equation in Sobolev spaces of negative indices, Duke Math. J., 71 (1993), 1-21.

[35] - On the generalized Benjamin-Ono equation, Trans. Amer. Math. Soc., 342 (1994), 155-172.

[36] - A bilinear estimate with applications to the KdV equation, J. Amer. Math. Soc., 9 (1996), 573-603.

[37] — Smoothing effects and local existence theory for the generalized nonlinear Schrödinger equations, Invent. Math., 134 (1998), 489-545.

[38] Kruzhkov, S. N. and Faminskii, A. V., Generalized solutions of the Cauchy problem for the Korteweg-de Vries equation, Math. USSR Sbornik, 48 (1984), 391-421.

[39] Mizohata, S., On some Schrödinger type equations, Proc. Japan Acad. Ser. A Math. Sci., 57 (1981), 81-84.

[40] Molinet, L., Saut, J. C. and Tzvetkov, N., Ill-posedness issues for the Benjamin-Ono and related equations, Preprint, Université de Paris-Sud, Orsay (2001).

[41] Nakamitsu, K., Analytic solutions of the nonlinear Schrödinger equation with localized $H^{1}$ data, unpublished manuscript (1997), Tokyo Denki University.

[42] Ono, H., Algebraic solitary waves in stratified fluids, J. Phys. Soc. Japan, 39 (1975), 1082-1091.

[43] Ponce, G., On the global well-posedness of the Benjamin-Ono equation, Diff. Integral Equations, 4 (1991), 527-542.

[44] Robbiano, L. and Zuily, C., Microlocal analytic smoothing effect for the Schrödinger equation., Duke Math. J., 100 (1999), 93-129.

[45] Sacks, B., Classical solutions of the Korteweg-de Vries equation for non-smooth initial data via inverse scattering, Comm. P.D.E., 10 (1985), 29-98.

[46] Saut, J. C., Sur quelques généralisations de l'équation de Korteweg-de Vries, J. Math. Pure Appl., 58 (1979), 21-61.

[47] Saut, J. C. and Temam, R., Remarks on the Korteweb-de Vries equation, Israel J. Math., 24 (1976), 78-87.

[48] Sjölin, P., Regularity of solutions to the Schrödinger equations, Duke Math. J., 55 (1987), 699-715.

[49] Tarama, S., Analyticity of the solution for the Korteweg-de Vries equation, Preprint. 
[50] Tom, M. M., Smoothing properties of some weak solutions of the Benjamin-Ono equation, Diff. Integral Equations, 3 (1990), 683-694.

[51] Tsutsumi, Y., The Cauchy problem for the Korteweg-de Vries equation with measure as initial data, SIAM J. Math. Anal., 20 (1989), 582-588.

[52] Ukai, S. Local solutions in Gevrey classes to the nonlinear Boltzmann equation without cutoff, Japan J. Appl. Math., 1 (1984), 141-156.

[53] Vega, L., The Schrödinger equation: pointwise convergence to the initial data, Proc. Amer. Math. Soc., 102 (1988), 874-878.

[54] Yosihara, H., Gravity waves on the free surface of an incompressible perfect fluid of finite depth, Publ. RIMS, Kyoto Univ., 18 (1982), 49-96. 